\title{
Predicting the near-wall velocity of wall turbulence using a neural network for particle image velocimetry
}

Cite as: Phys. Fluids 32, 115105 (2020); https://doi.org/10.1063/5.0023786

Submitted: 02 August 2020 . Accepted: 13 October 2020 . Published Online: 03 November 2020

(D) Hongping Wang (王洪平), (D) Zixuan Yang (杨子轩), Binglin Li (李秉霖), and (D Shizhao Wang (王士召)

\section{COLLECTIONS}

EP This paper was selected as an Editor's Pick
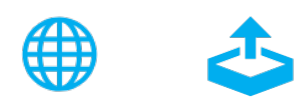

\section{ARTICLES YOU MAY BE INTERESTED IN}

Artificial neural network-based nonlinear algebraic models for large eddy simulation of turbulence

Physics of Fluids 32, 115101 (2020); https://doi.org/10.1063/5.0025138

Deconvolutional artificial neural network models for large eddy simulation of turbulence Physics of Fluids 32, 115106 (2020); https://doi.org/10.1063/5.0027146

Two-stage growth mode for lift-off mechanism in oblique shock-wave/jet interaction Physics of Fluids 32, 116105 (2020); https://doi.org/10.1063/5.0022449

\section{Physics of Fluids GALLERY OF GOVERS}




\title{
Predicting the near-wall velocity of wall turbulence using a neural network for particle image velocimetry
}

\author{
Cite as: Phys. Fluids 32, 115105 (2020); doi: 10.1063/5.0023786 \\ Submitted: 2 August 2020 - Accepted: 13 October 2020 • \\ Published Online: 3 November 2020
}

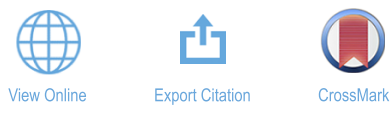

\begin{abstract}
Hongping Wang (王洪平), (D) Zixuan Yang (杨子轩), (D) Binglin Li (李秉霖), and Shizhao Wang (王士召)
\end{abstract}
AFFILIATIONS

The State Key Laboratory of Nonlinear Mechanics, Institute of Mechanics, Chinese Academy of Sciences, Beijing 100190, China

a) Author to whom correspondence should be addressed: wangsz@lnm.imech.ac.cn

\begin{abstract}
Near-wall velocity prediction for wall-bounded turbulence is useful for constructing a wall model and estimating dissipation and wall shear stress. A convolutional neural network is developed to improve the near-wall velocity prediction and spatial resolution for wall-bounded turbulent velocity fields obtained using particle image velocimetry (PIV). To establish the relationship between the low-resolution and highresolution fields, this machine learning model is trained on a synthetic PIV dataset generated based on velocity fields obtained from the direct numerical simulation of turbulent channel flows at $R e_{\tau}=1000$. Using a test dataset with a higher Reynolds number of $R e_{\tau}=5200$, the performance of this model is assessed in terms of instantaneous fields, error analysis, velocity statistics, and energy spectra. The influences of the interrogation window, image resolution, and particle concentration on the performance of this network are also considered. We further apply this network to practical PIV data from a turbulent boundary layer at $R e_{\tau}=2200$ to assess the network performance under real experimental conditions. The results indicate that the proposed machine-learning-based model can predict missing near-wall velocity fields and enhance the spatial resolution of PIV fields, but the accuracy for Reynolds shear stress prediction needs to be further improved. The presented approach shows the potential ability to predict the near-wall instantaneous velocity of high-Reynolds-number turbulence from low-Reynolds-number flow fields.
\end{abstract}

Published under license by AIP Publishing. https://doi.org/10.1063/5.0023786

\section{NOMENCLATURE}

$B$ batch size

C number of velocity components

$d_{p} \quad$ particle image diameter (pixels)

$D_{R} \quad$ digital imaging resolution (wall units/pixel)

$H \quad$ height of the velocity field

ppp particles per pixel

$R e_{\tau}$ friction Reynolds number

$u \quad$ streamwise velocity

$v \quad$ wall-normal velocity

$W \quad$ width of the velocity field

$x \quad$ streamwise coordinate

$y \quad$ wall-normal coordinate

$\Delta W \quad$ interrogation window size (pixels)

\section{Greek symbols}

$\delta$ root-mean-square error

\author{
Abbreviations \\ 3D3C three-dimensional and three-component \\ DNN deep neural network \\ DNS direct numerical simulation \\ IW interrogation window \\ LES large eddy simulation \\ ML machine learning \\ PIV particle image velocimetry \\ WU wall unit
}

\section{INTRODUCTION}

Turbulence is a ubiquitous natural phenomenon and is also crucial to many engineering applications. For the most common wall-bounded turbulence, a strong shear region is generated in the near-wall region due to the no-slip condition at the wall surface. This shear region is related to turbulence production, dissipation, 
turbulent kinetic energy, and drag force and has been studied over the past several decades (Pope, 2000; Marusic et al., 2010b; Mathis et al., 2011; and de Giovanetti et al., 2016). Understanding and predicting the behavior of wall turbulence is still a challenging goal for both computational fluid dynamics and experimental fluid dynamics. In the field of computational fluid dynamics, the prediction of near-wall velocity can be used as a wall model for large eddy simulation (LES) and Reynolds-averaged Navier-Stokes (RANS) numerical simulations (Karimpour and Venayagamoorthy, 2013 and Stanislas, 2017). In the field of experimental fluid dynamics, as stated by Mathis et al. (2011), accurate turbulence measurements in the near-wall region become very challenging at high Reynolds numbers due to the decreasingly small size of this region. For particle image velocimetry (PIV), which is a widely used and powerful tool to analyze turbulence properties and visualize coherent structures (Christensen and Adrian, 2001; Adrian, 2005; Hutchins and Marusic, 2007; Westerweel et al., 2013; and Deng et al., 2018), the spatial resolution issue is inevitable since PIV involves spatial filtering over an interrogation window (IW). Especially, in the near-wall region, on the one hand, PIV is incapable of resolving the velocity very close to the wall due to the IW, while on the other hand, the root mean square (rms) vorticity profile and Reynolds stresses are systematically underestimated to $\sim 50 \%$ of the values found through direct numerical simulation (DNS) (Atkinson et al., 2013 and Schneiders et al., 2017). Therefore, if the near-wall turbulence cannot be accurately resolved, a prediction model is highly desired, especially, for velocity-gradient-based wall shear stress estimation (Wang et al., 2020) and flow controls (Abbassi et al., 2017) at high Reynolds numbers.

In the near-wall region of wall turbulence, the streaks observed by Kline et al. (1967) and quasi-streamwise vortices are the dominant coherent structures (Smits et al., 2011). The inner energy peak is located at the wall-normal location $y^{+}=15$ (the superscript + denotes a normalization with inner scales), and the dominant streamwise and spanwise wavelengths, which are scaled to viscous wall units (WUs), are $\sim 1000$ and 100, respectively (Marusic et al., 2010a). These values are nearly invariant at different Reynolds numbers. The numerical simulations by Jiménez and Pinelli (1999) and Schoppa and Hussain (2002) indicated the existence of a selfsustaining near-wall cycle that is a local phenomenon at the nearwall region and is not influenced by the outer flow. Furthermore, the outer large-scale structures indeed have a footprint in the nearwall region, which is consistent with the attached-eddy hypothesis proposed by Townsend (1976). All the above findings indicate that the near-wall organization of wall-bounded turbulence may be universal and can be predicted through mathematical or physical or data-driven approaches. Mathis et al. (2011) investigated the correlation of the inner and outer signals and found that the outer structures affect the near-wall turbulent fluctuations by superposition and modulation. A concise algebraic outer-inner model was proposed to predict the near-wall turbulence with only large-scale information from the outer boundary layer region. A prediction method based on the minimal flow unit (MFU) was proposed for the three velocity components (Yin et al., 2018). The MFU is replicated periodically in the streamwise and spanwise directions to model the near-wall flow structures. For experimental fluid dynamics, near-wall velocity prediction can be used to estimate or correct the dissipation and wall shear stress in the near-wall region. Zaripov et al. (2019) stated that it still suffers from measurement error in the near-wall region $\left(y^{+}<20\right)$, yielding an overestimated dissipation rate. The vortex-incell plus (VIC+) method, which uses the vorticity transport equation to optimize the particle tracking velocimetry (PTV) measurements, is applied to resolve the dissipation in a turbulent boundary layer (Schneiders et al., 2017). To accurately calculate the wall shear stress, an elongated rectangle IW is adopted to enhance the resolution in the wall-normal dimension (Wang et al., 2020). They also reported that the number of particles below $y^{+}=30$ is quite smaller than the commonly accepted value used in the conventional PIV algorithm (Raffel et al., 2018). The sparsity of the particles in the near-wall region implies that the near-wall velocity estimated by PIV is unreliable. In summary, the near-wall velocity prediction is possible and necessary.

According to Stanislas (2017), it has not yet been possible to make use of this wall turbulence organization to improve any near-wall turbulence model. Therefore, a data-driven approach, i.e., machine learning (ML), may be a possible way to accurately predict the near-wall velocity. As far as we know, recently, the machine learning has been at least applied to the turbulence model and velocity supersolution. In the review papers by Duraisamy et al. (2019) and Zhang et al. (2019a), data-driven approaches combined with physical constraints are exploited to yield useful predictive turbulence models. Zhou et al. (2019) used a neural network to develop a new subgrid-scale model for LES of isotropic turbulent flows. The performance of the proposed neural network model is better than that of the Smagorinsky model and dynamic Smagorinsky model. Yang et al. (2019) used physics-informed neural networks to predict the wall-shear stress for wall-modeled LES. The results indicate that the network models outperform the conventional equilibrium wall model in a nonequilibrium flow. Zhang et al. (2019b) used a deep neural network (DNN) to predict the Reynolds stresses of channel flow at different Reynolds numbers. The prediction accuracy of DNN is higher than that of the LES model. Zhu et al. (2019) directly built the relationship between the turbulent eddy viscosity and the mean flow variables using the neural network for subsonic flows around airfoils and completely replaced the original partial differential equation model. Zhang et al. (2020) improved RANS predictions with various data assimilation techniques by incorporating DNS velocity data. A symmetrical DNN is proposed to realize the reconstruction and prediction of the flow field structure in the supersonic cascade channel under complicated and changing working conditions (Li et al., 2020). Renganathan et al. (2020) used DNNs to construct reduced-order models for the fast exploration of design space for diverse engineering applications. Zhang and $\mathrm{Ma}$ (2020) discovered the governing equations and transport coefficients using the ML method. This data-driven approach provides a new way to establish the governing equations for nonequilibrium flows and complex fluids. Inspired by the achievement of image superresolution in the field of computer vision (Dong et al., 2014; Lim et al., 2017; and Ledig et al., 2017), the super-resolution reconstruction of turbulent flows using DNNs has received increasing research attention (Fukami et al., 2019; Deng et al., 2019; and Liu et al., 2020) in the fluid mechanics field. Using flow fields from high-fidelity simulations and experiments, high-resolution fields can be accurately predicted from low-resolution fields using a DNN. This technique can be potentially used to accurately estimate the turbulent dissipation rate for PIV fields (Xu and Chen, 2013). More discussions about 
the applications of ML on understanding, modeling, optimizing, and controlling fluid flows can be found in the review paper by Brunton et al. (2020).

In the present work, a DNN is used to predict the near-wall velocity of PIV measurements for wall turbulence. The predicted velocity can be used to estimate dissipation and wall shear stress. Near-wall prediction is more challenging than the super-resolution reconstruction of flow fields because the former can be regarded as an extrapolation in mathematics. There are two objectives in this research. The first is to predict the near-wall velocity in the region where the PIV algorithm is invalid. The second objective is to enhance the spatial resolution of PIV fields by using a DNN to enrich the dynamics at small scales that are filtered out by PIV. To achieve these objectives, we use a modified version of a state-of-theart network known as an enhanced deep super-resolution network (EDSR) (Lim et al., 2017) to construct the relationship between the low-resolution and high-resolution fields. The rest of this paper is organized as follows: In Sec. II, we first introduce the network architecture used in the present work and describe the generation of the training and test datasets. In Sec. III, the performance of the network is assessed based on the test dataset in terms of instantaneous fields, error analysis, velocity statistics, and energy spectra. In Sec. IV, we present the application of the network to process data from a real experiment involving a turbulent boundary layer. Finally, we conclude in Sec. V.

\section{METHOD}

\section{A. The structure of the neural network}

The network used in the present work is a kind of convolutional neural network; therefore, we will first introduce the frequently used convolutional layer. A convolutional layer contains a set of filters whose weights need to be learned. Each filter is convolved with the input data by sliding the filter across the width and height of the input (Ke et al.,2018). Figure 1 gives a schematic diagram of the 2D convolutional layer with one filter kernel. A convolution operation

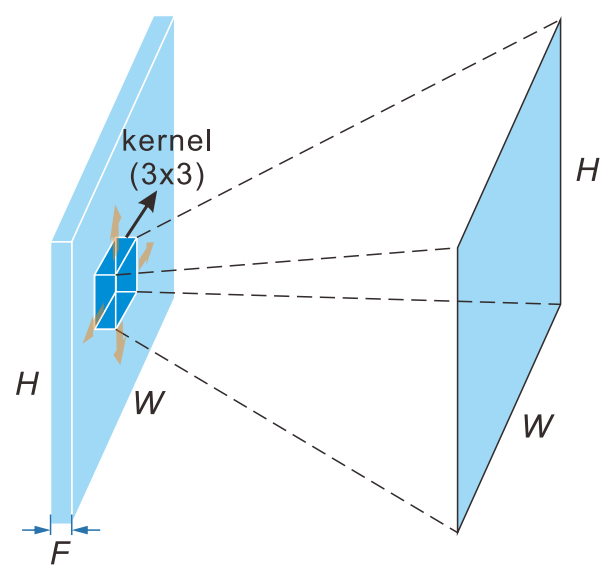

FIG. 1. Schematic diagram of a two-dimensional (2D) convolutional layer with one filter kernel. is applied to the input array with dimensions of $H \times W \times F$, where $H$ and $W$ are the height and width of the input array and $F$ is the number of features. The size of the filter kernel is $3 \times 3 \times F$. In this work, the output size is still the same as the input, i.e., $H \times W \times 1$ for one filter kernel.

Figure 2 shows the architecture of the network used in the present work, which is a modified version of the EDSR architecture proposed by Lim et al. (2017). The proposed super-resolution neural network for 2D PIV (PIV2DSR) is constructed for a fixed upsampling factor of four, which means that the size of the output velocity field is four times that of the input velocity field. The network consists of convolutional layers and residual blocks (Lim et al., 2017). The input is a two-component velocity field with dimensions of $B$ $\times H \times W \times C$, where $B$ is the batch size and $C$ is the number of components. In the present work, the parameter $C$ is equal to 2 . The first layer performs convolution with 64 different $3 \times 3 \times 2$ filter kernels, yielding feature maps with dimensions of $H \times W \times 64$ for each velocity field. The second layer is also a convolutional layer with 64 kernels. The third layer consists of six residual blocks. In each residual block, the input is summed with the residual generated from a chain consisting of a convolutional layer, a rectified linear unit layer, another convolutional layer, and a multiplication layer. The rectified linear unit layer is the most commonly used activation function in deep learning and helps a model account for nonlinearity. The rectified linear unit function returns 0 if the input is negative, while it returns the same value if the input is positive. The rectified linear unit function can be written as $f(x)=\max (0, x)$ (Glorot et al, 2011). A constant factor of 0.2 is adopted in the multiplication layer to scale the residual (Lim et al., 2017). The fourth layer is a convolutional layer with 32 features, and the dimensions of the output after this convolutional layer are $H \times W \times 32$. The first layer is additionally convolved by 32 features, and then, the result is added to the result from the fourth convolutional layer. Finally, the summed result, with dimensions of $B \times H \times W \times 32$, is upscaled four times for a high-resolution field with dimensions of $B \times 4 H \times 4 W \times 2$ using a shuffling operator (Shi et al., 2016).

Through suitable training, PIV2DSR can construct the relationship between an input dataset $\boldsymbol{x}$ and the desired output (groundtruth) dataset $y$. We use $F$ and $\boldsymbol{w}$ to denote the network model and the weights in this model, respectively. Thus, in the present case, $x$ and $F(\boldsymbol{x} ; \boldsymbol{w})$ represent the input low-resolution PIV data and the corresponding output data with 4 times higher resolution, respectively. The configuration shown in Fig. 2 yields a total of 518208 weights; these weights only come from the filter kernels and are independent of the resolution and number of velocity fields. Taking Fig. 1 as an example, the number of unknown weights needing to be trained is $3 \times 3 \times F$. These weights should be optimized to minimize the mean square error (MSE) between the output data $F(x ; \boldsymbol{w})$ and the ground-truth data $y$ as follows:

$$
\boldsymbol{w}=\operatorname{argmin}_{\boldsymbol{w}}\left\{\frac{\|\boldsymbol{y}-F(\boldsymbol{x} ; \boldsymbol{w})\|_{2}^{2}}{N}\right\},
$$

where the parameter $N=B \times 4 H \times 4 W \times 2$ represents the total spatial number of outputs in a batch. The PIV2DSR model can be used for prediction once the weights $w$ have been determined through the training process. 


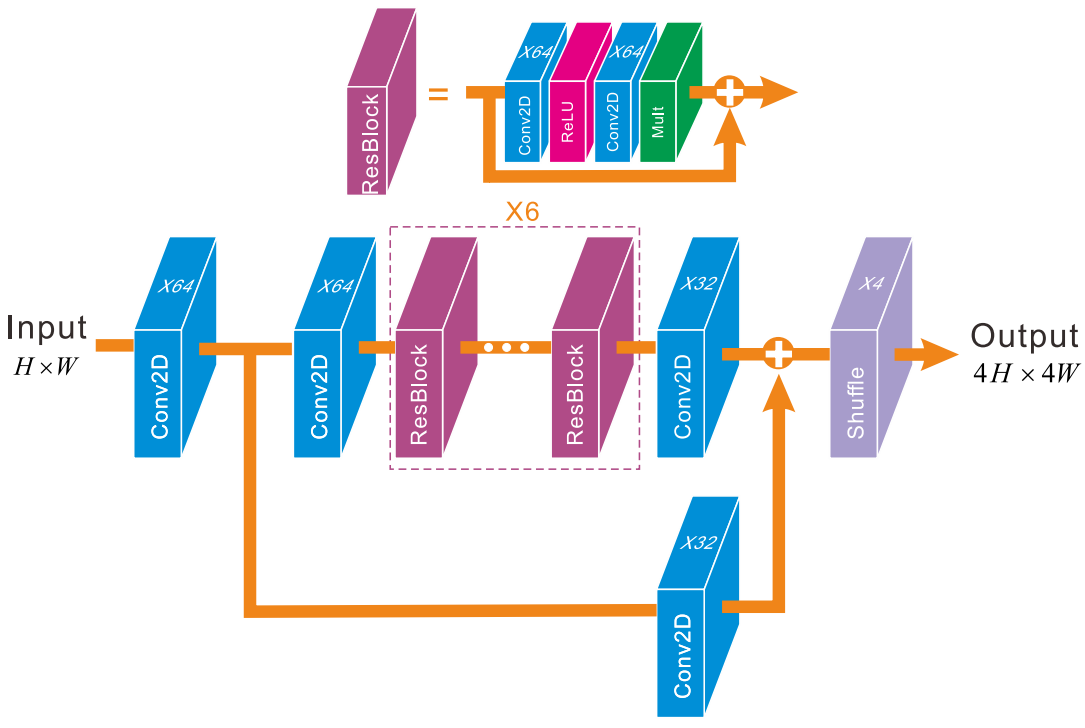

FIG. 2. The architecture of the proposed network, PIV2DSR.

\section{B. Dataset generator}

Before introducing the dataset generation, we will first elucidate all the parameters used in dataset generation, as listed in Table I. The streamwise and wall-normal directions are denoted by $x$ and $y$, respectively, and the corresponding velocities are denoted by $u$ and $v$, respectively. The velocity $u$ can be decomposed into a mean value $U=\langle u\rangle$ and a fluctuation $u^{\prime}$ such that $u=U+u^{\prime}$. The parameters listed in Table I include the streamwise length $\left(L_{x}\right)$ and image size $\left(M_{x}\right)$, the wall-normal height $\left(L_{y}\right)$ and image size $\left(M_{y}\right)$, the number of particles per pixel $(p p p)$, the digital imaging resolution $\left(D_{R}\right)$, and the particle image diameter $\left(d_{p}\right)$. The parameters $L_{x}$ and $L_{y}$ are normalized to WUs, and for convenience, $D_{R}$ is expressed in WUs/pixel rather than in pixels/WU (Scarano, 2013).

The training dataset is generated via the DNS of turbulent channel flows with a Reynolds number of $R e_{\tau}=1000$. We prepared 4000 independent $2 \mathrm{D}$ velocity fields in the $x-y$ plane. The synthetic particle images are generated in accordance with the EUROPIV synthetic image generator proposed by Lecordier and Westerweel (2004). The generator first randomly picks a value for $D_{R}$ from the candidates of
$0.5,1.0$, and 1.5 and then calculates the field of view (FOV) as $\left(L_{x}\right.$, $\left.L_{y}\right)=\left(1000 D_{R}, R e_{\tau}\right)$. Synthetic PIV images were generated, beginning at $y^{+}=0$, to cover the whole turbulent boundary layer. The superscript " + " denotes the dimensionless with WU based on the friction velocity $u_{\tau}$ and the kinematic viscosity $v$. The parameter $p p p$ was fixed to 0.05 . The positions of the particles in A and B exposures are determined as $\left(x_{p}-u_{p} / 2, y_{p}-v_{p} / 2\right)$ and $\left(x_{p}+u_{p} / 2, y_{p}\right.$ $\left.+v_{p} / 2\right)$, respectively, where $\left(x_{p}, y_{p}\right)$ is the random location of the particle and $\left(u_{p}, v_{p}\right)$ is the velocity obtained by interpolating the DNS velocity fields. The particle intensity was calculated by integrating the 2D Gaussian intensity distribution over the pixel area with an image particle diameter of 2 pixels and a standard deviation of 0.2 pixels (Sciacchitano et al., 2013). The intensity values of the particle images were converted into 8-bit unsigned integers. No image noise is considered in the present work.

The PIV velocity was estimated using a three-pass window deformation iterative multigrid (WIDIM) scheme (Scarano and Riethmuller, 2000 and Scarano, 2002). Five different IW sizes $\Delta W$ of $16 \times 16$ pixels $^{2}, 24 \times 24$ pixels $^{2}, 32 \times 32$ pixels $^{2}, 40 \times 40$ pixels $^{2}$, and $48 \times 48$ pixels $^{2}$ with $75 \%$ overlap were utilized. Note that the IW

TABLE I. Parameters used in dataset generation.

\begin{tabular}{llll}
\hline \hline & & Training & Test \\
\hline Reynolds number & $R e_{\tau}$ & 1000 & 5200 \\
Imaging resolution & $D_{R}(\mathrm{WU}$ s/pixel) & $0.5,1.0,1.5$ & $0.5,0.75,1.0,1.25,1.5$ \\
Image size & $M_{x} \times M_{y}$ (pixels) & $1000 \times \frac{R e_{\tau}}{D_{R}}$ & $2048 \times 512$ \\
Particle diameter & $d_{p}$ (pixels) & $2 \pm 0.2$ & $2 \pm 0.2$ \\
Particles per pixel & $p p p$ & 0.05 & $0.01,0.03,0.05,0.07,0.09$ \\
Streamwise length & $L_{x}$ (WUs) & $1000 D_{R}$ & $2048 D_{R}$ \\
Wall-normal height & $L_{y}(\mathrm{WUs})$ & $R e_{\tau}$ & $512 D_{R}$ \\
Interrogation window & $\Delta W$ (pixels) & $16,24,32,40,48$ & $16,24,32,40,48$ \\
Number of fields & $K$ & 16000 & 500 \\
\hline \hline
\end{tabular}


size used for the first pass was larger than the final IW size to ensure robust flow fields. The cross correlation maps for displacement estimation were calculated using a Fast Fourier Transform (FFT)-based approach, and the subpixel displacements were interpolated by a three-point Gaussian function (Raffel et al., 2018). Outliers were detected using the normalized median test (Westerweel and Scarano, 2005), and the missing vectors were filled using linear interpolation. An average filtering with dimensions of $3 \times 3$ was then applied to the final fields to further reduce the measurement noise. The velocities computed via PIV exhibit very high bias errors and random errors near the wall due to the strong velocity gradient. Thus, the unreliable vectors at $y^{+} \leq 20$ were removed from the PIV fields. Then, all velocity fields were linearly extrapolated to a regular square mesh beginning at $y^{+}=0$ by enforcing a zero velocity at the wall, with a grid spacing equal to that of the original PIV velocity field. Subsequently, the interpolated velocity fields were divided into many small blocks with dimensions of $32 \times 32$ and an overlap factor of $50 \%$ to obtain the inputs to the network. Accordingly, the parameters $H$ and $W$ for the training set were 32 and 32, respectively. Most of the small blocks are off the wall, i.e., the bottom position $y^{+}>0$. The number of these blocks is denoted as $N_{\text {off }}$. Only a small part of the blocks begin at $y^{+}=0$, and the number is denoted as $N_{o n}$. To enhance the network performance near the wall, the former was part randomly removed from the dataset. Therefore, the ratio of the near-wall velocity fields estimated as $N_{\text {on }} /\left(N_{\text {off }}+N_{\text {on }}\right)$ was increased to $\sim 60 \%$. Figure 3 shows the entire flow chart for the training of the PIV2DSR model. The step-by-step procedure for synthetic PIV field generation is described as follows:

1. Initialize the parameters listed in Table I. The values of the parameters $D_{R}$ and $\Delta W$ are randomly selected from among the candidates. The image size is set to $1000 \times \frac{R e_{\tau}}{D_{R}}$ pixels $^{2}$.

2. Read a reference velocity field from the corresponding DNS dataset.

3. Generate particle image pairs in accordance with the parameters and the DNS velocity field. The center difference

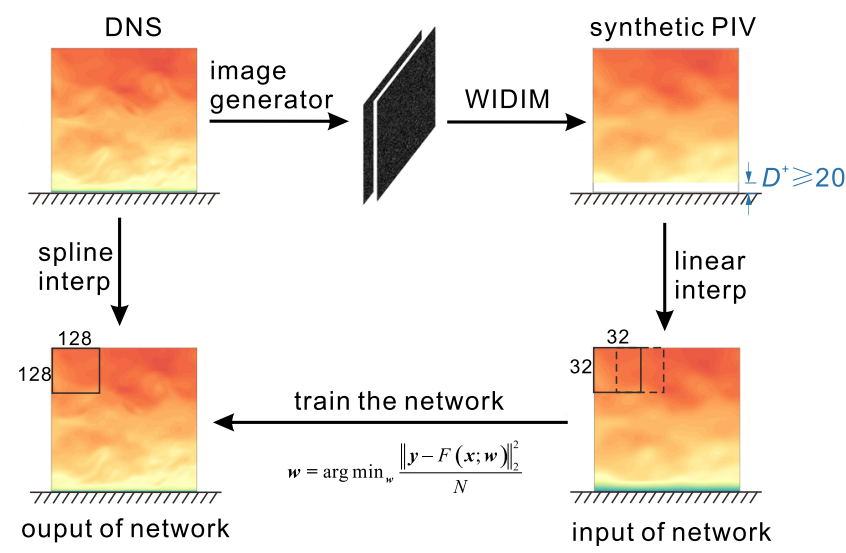

FIG. 3. Flow chart for PIV2DSR model training. In this figure, the parameter $D^{+}$ represents the distance from the bottom boundary of the PIV field to the wall. The input, with dimensions of $32 \times 32$, is upscaled to output with dimensions of 128 $\times 128$ by the network. scheme is adopted to determine the positions of the particles.

4. Estimate the velocity field from the image pairs using WIDIM and remove the vectors for $y^{+} \leq 20$.

5. Interpolate the synthetic PIV field over the mesh containing the region starting at the wall, $y^{+}=0$, using the same vector spacing as that of the original PIV field.

6. Divide the interpolated PIV field into small blocks with dimensions of $32 \times 32$ and $50 \%$ overlap.

7. Interpolate the reference DNS velocity field to a mesh of 4 times higher resolution corresponding to the mesh used in step 6 to obtain the ground truth.

8. Repeat steps 1-7 until the number of synthetic PIV fields obtained for training is greater than 16000 .

To validate the performance of PIV2DSR, a test dataset completely independent of the training dataset was generated via the DNS of turbulent channel flows at $R e_{\tau}=5200$. The DNS data were downloaded from the Johns Hopkins Turbulence Databases (Perlman et al., 2007; Li et al., 2008; and Graham et al., 2016). Five hundred 2D $x-y$ slices of two-component velocity data were used to generate particle image pairs. As listed in Table I, $p p p$ was varied from 0.01 to 0.09 in intervals of 0.02 and $D_{R}$ was varied from 0.5 to 1.5 in intervals of 0.25 . The positions of the particles were randomly distributed in each image with a resolution of 2048 $\times 512$ pixels $^{2}$. The PIV velocities were also estimated using the same configuration used to generate the training dataset. Five hundred velocity fields for every case were generated for performance validation, resulting in a total of 62500 velocity fields in the test dataset.

\section{Results of model training}

The PIV2DSR was coded based on the open-source ML platform TensorFlow v2.1.0 in Python and shared on https://github.com/ hpwang87/PIV2DSR. In general, there is no minimum computational capability requirement to use a common central processing unit (CPU) for TensorFlow. However, it is best to use a highperformance NVIDA graphics processing unit (GPU) card with compute unified device architecture (CUDA) to speed up the training process. The TensorFlow supports the GPU with the compute capability 3.5 and higher. In our work, the network was trained and tested on a workstation equipped with an Intel Xeon W2245 3.90 $\mathrm{GHz}$ CPU and a single NVIDA Quadro RTX $5000 \mathrm{GPU}$ with $16 \mathrm{~GB}$ RAM. The whole training set was fed into PIV2DSR to construct the relationship between the low-resolution PIV data and the highresolution reference DNS data. Approximately $10 \%$ of the data were reserved as a validation dataset to check the generalization ability of the network. The Adam optimizer was used to update the weights with a learning rate of $1 \times 10^{-4}$ and a batch size of 128 . The training process was stopped after 1000 epochs, $\sim 2.5 \mathrm{~h}$, and the loss as a function of the number of epochs is shown in Fig. 4. The training loss and validation loss converged to $\sim 0.045$ and 0.085 , respectively. The training process was stopped because the validation loss could no longer be reduced. The relative error for the maximum mean streamwise velocity is $\sim 0.65 \%$ corresponding to a mean error of 0.13 WUs. The performance of the PIV2DSR model is quantitatively analyzed based on the test dataset in Sec. III. 


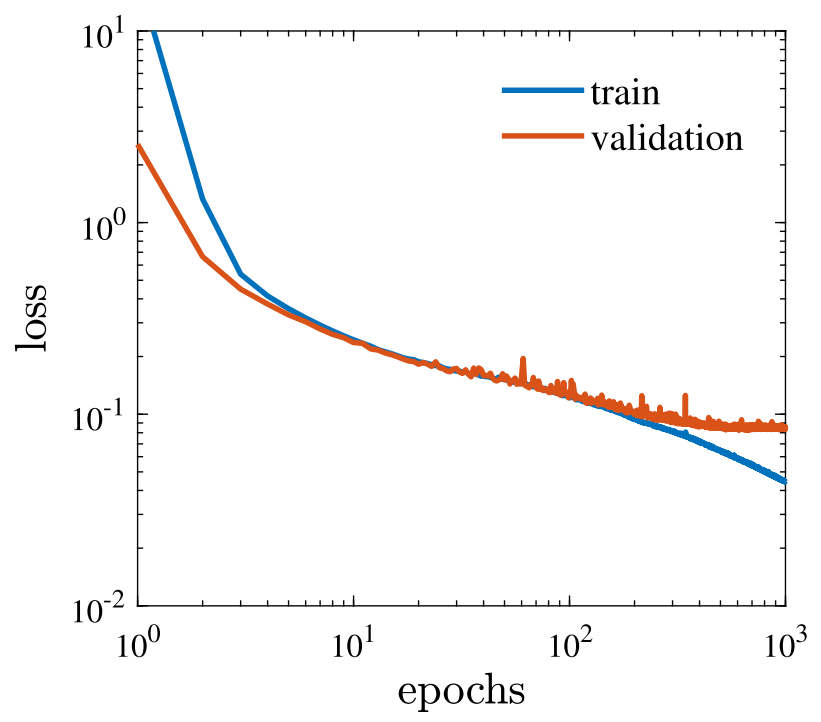

FIG. 4. Loss as a function of the number of epochs on the training and validation sets.

\section{NUMERICAL ASSESSMENTS ON THE TEST DATASET}

The validity of PIV2DSR was examined by using the test dataset of turbulent channel flows at $R e_{\tau}=5200$. The instantaneous flow fields, error distribution, velocity statistics, and energy spectra are studied in this section. The effects of the IW, digital imaging resolution, and particle seeding concentration on the network model are also evaluated.

\section{A. Instantaneous flow fields}

The validity of velocity reconstruction using the trained PIV2DSR model is first examined by visually inspecting the instantaneous velocity fields, as shown in Fig. 5. The results for IW sizes of $\Delta W=48$ pixels and 16 pixels are displayed in Figs. 5(a), 5(b) and in Figs. 5(c), 5(d) respectively. The left panels present the contour maps of the streamwise velocity fields $u^{+}$, and the right panels present the contour maps of the vorticity $\omega^{+}$. The $p p p$ and $D_{R}$ values are 0.05 and 1 , respectively. The original PIV field, the linearly interpolated PIV field (the input to the network), the predicted high-resolution field (the output of the network), and the reference DNS field are displayed from top to bottom in each panel. The $y$-axis is presented on a log scale to clearly illustrate the predicted near-wall velocity field. It is obvious that the near-wall velocity is missing in the PIV flow fields, and the bottom boundaries $D^{+}$for $\Delta W=16$ and 48 are 24.5 and 28.5, respectively. Regardless of whether the input velocity field is coarse or fine, it is obvious from Figs. 5(a)-5(d) that the velocity field resolution is enhanced in the region of $y^{+} \geq D^{+}$. Meanwhile, the proposed PIV2DSR has the ability to predict the unknown flow field in the near-wall region. In general, the output high-resolution field is closer to the DNS data even in the near-wall region without PIV data.

\section{B. Error analysis}

The root-mean-square error between the velocity obtained by PIV2DSR, which is denoted by $\mathbf{U}_{F}$, and the ground truth $\mathbf{U}_{e}$ is computed as follows:

$$
\delta=\sqrt{\frac{1}{N} \sum_{x \in \Omega}\left\|\mathbf{U}_{F}(x)-\mathbf{U}_{e}(x)\right\|_{2}^{2}},
$$

where the parameter $N$ is the number of vectors in the computation domain $\Omega$.

Figure 6(a) presents the error $\delta$ as a function of $y^{+}$for $p p p=0.05$ and $D_{R}=1$. The different colors represent different sizes of the IW. The errors of the original PIV and PIV2DSR velocities are shown on the top and bottom, respectively. As one might expect, the PIV error exhibits an increasing trend as $y^{+}$decreases. This is caused by the rapidly increasing streamwise velocity gradient observed in wallbounded turbulence. Taking the results for $\Delta W=16$ as an example, the root-mean-square error increases from 0.2 in the outer layer to 0.44 at $y^{+}=24.5$. We believe that the error will continue to increase rapidly in the near-wall region, as shown in the paper of Atkinson et al. (2011). Moreover, a smaller IW can yield a lower error due to the ideal nature of the simulation in which image noise is not considered. The results are consistent with those of Atkinson et al. (2011). In contrast, the error of PIV2DSR is generally much lower than that of PIV and exhibits a peak at $y^{+} \approx 10$. The $\delta$ value for $\Delta W=16$ pixels is $\sim 0.12$ in the outer layer and 0.32 at $y^{+}=24.5$. Meanwhile, the error of PIV2DSR is also reduced as $\Delta W$ decreases. The error values $\delta$ under different $D_{R}$ conditions with a fixed IW size of $\Delta W=32$ pixels are given in Fig. 6(b). It can be seen from Fig. 6(b) that both the PIV and PIV2DSR errors are reduced as $D_{R}$ decreases, while the error of PIV2DSR is much lower than that of PIV. Figures 6(a) and 6(b) provide the insight that the error of PIV2DSR is reduced as the PIV velocity resolution increases. The availability of more information from the flow motion is helpful to improve the prediction accuracy of the network.

For PIV, $p p p$ can influence the accuracy of velocity estimation. The presence of fewer particles in the IW will result in greater random noise due to the incredible correlation peak. The average errors across the measured boundary layer under different $\triangle W, D_{R}$, and $p P p$ values are presented in Table II. Here, the errors of both PIV $\left(\delta_{P I V}\right)$ and PIV2DSR $\left(\delta_{P I V 2 D S R}\right)$ were calculated over all velocity fields above $y^{+}=D^{+}$. The errors are seen to be more sensitive to $\triangle W$ and $D_{R}$ than to $P p p$ within the range of our simulations. This is because the particle images were ideally simulated without considering experimental noise. Smaller errors can be observed in the case of PIV2DSR for various values of $p p p$.

Under the condition $p p p=0.05$, the mean error across the boundary layer as a function of $\Delta W$ and $D_{R}$ is presented in Fig. 7. The absolute error contour maps of $\delta_{P I V}$ and $\delta_{P I V 2 D S R}$ are displayed in Figs. $7(\mathrm{a})$ and $7(\mathrm{~b})$, respectively. The error $\delta_{P I V}$ decreases as the image resolution $D_{R}$ and $\Delta W$ decrease. An optimal $\Delta W$ can be identified when $D_{R}$ is less than 0.75 . As seen by comparing Figs. 7(a) and $7(\mathrm{~b})$, the error $\delta_{P I V 2 D S R}$ is much smaller than $\delta_{P I V}$. The relative error reduction of PIV2DSR, estimated as $\left(\delta_{P I V 2 D S R}-\delta_{P I V}\right) / \delta_{P I V}$ across the measured boundary layer, is shown in Fig. 7(c) for $p p p$ $=0.05$. A visual inspection of the contours reveals that the degree of error reduction increases as $D_{R}$ decreases but is insensitive to $\Delta W$. 


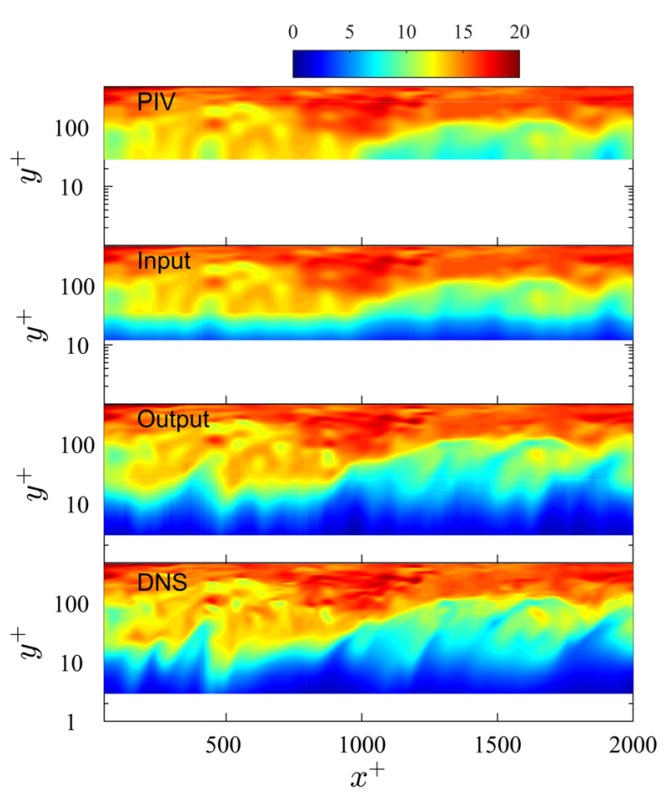

(a)

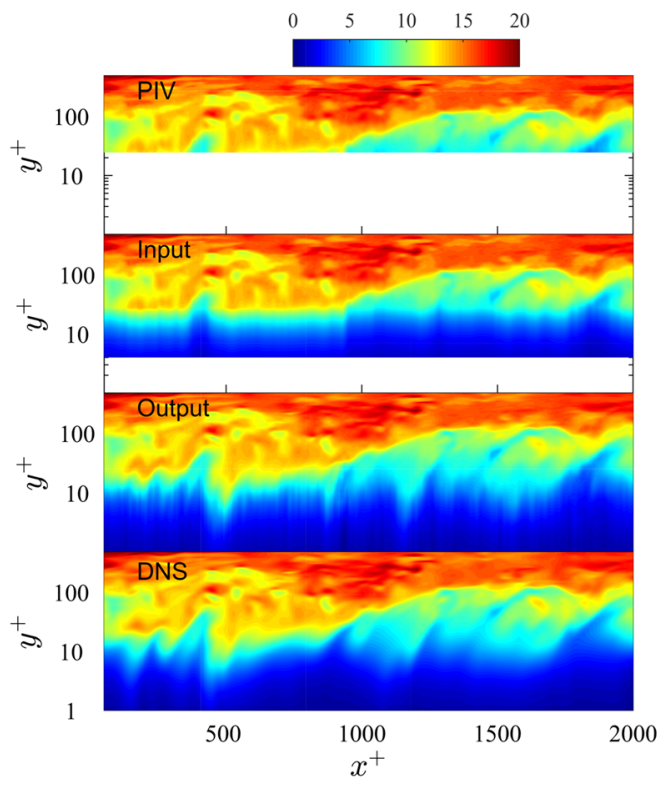

(c)

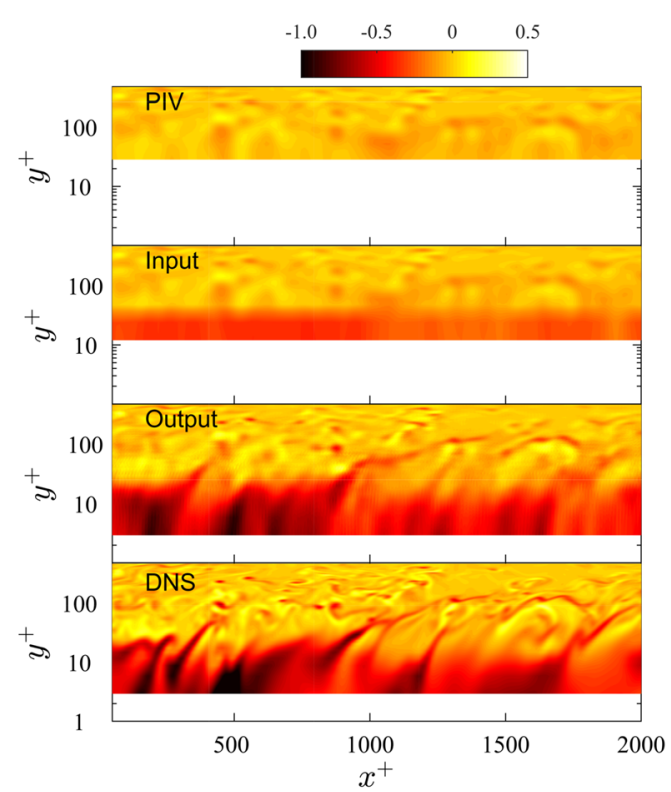

(b)

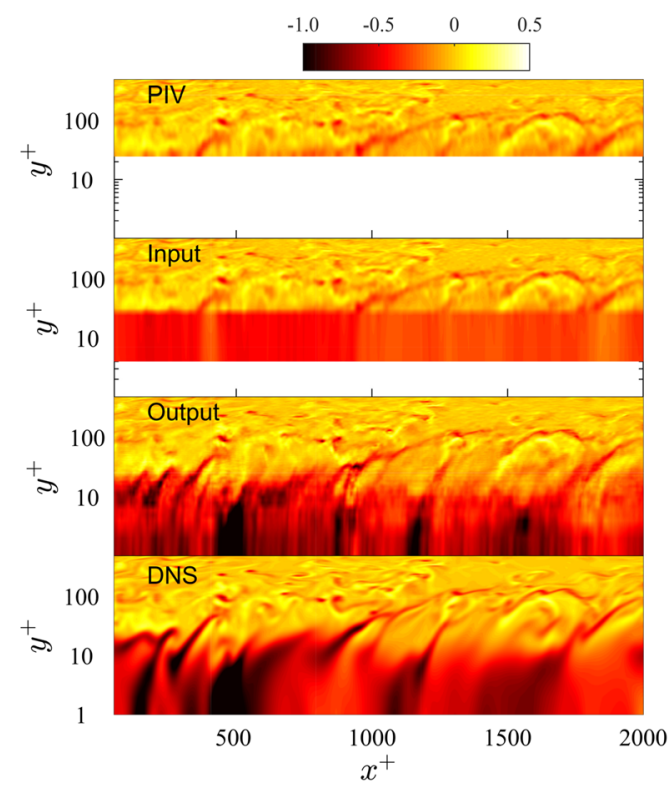

(d)

FIG. 5. Comparison of the instantaneous fields at $R e_{\tau}=5200$ with $p p p=0.05$ and $D_{R}=1$. In each panel, the original PIV field, the linearly interpolated PIV field (the input to the network), the predicted high-resolution field (the output of the network), and the reference DNS field are displayed from top to bottom. Panels (a) and (b) show the results obtained with $\Delta W=48$ pixels, and panels (c) and (d) show the results obtained with $\Delta W=16$ pixels. Panels (a) and (c) show the contour maps of the streamwise velocity $u^{+}$, and panels (b) and (d) show the contour maps of the vorticity $\omega^{+}$.

After processing with PIV2DSR, the PIV error can be reduced by $\sim 20 \%$ when $D_{R}=1.5$ and by $\sim 30 \%$ when $D_{R}=0.5$. To simplify the discussion, only three cases, indicated by red dots, are considered to perform the further statistical and spectral analyses presented in Secs. III C and III D, respectively.
Based on this error analysis, we would like to offer two remarks on the performance of PIV2DSR. First, the neural network model maintains a good generalization ability across different values of the experimental parameters $\left(\Delta W, D_{R}\right.$, and $\left.p p p\right)$. A real experimental assessment based on a turbulent boundary layer will be 


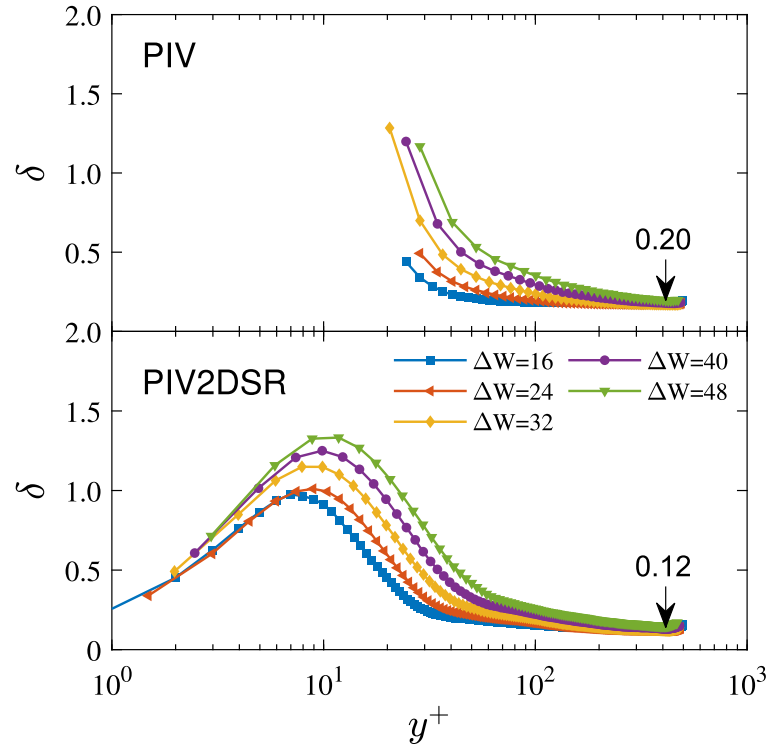

(a)

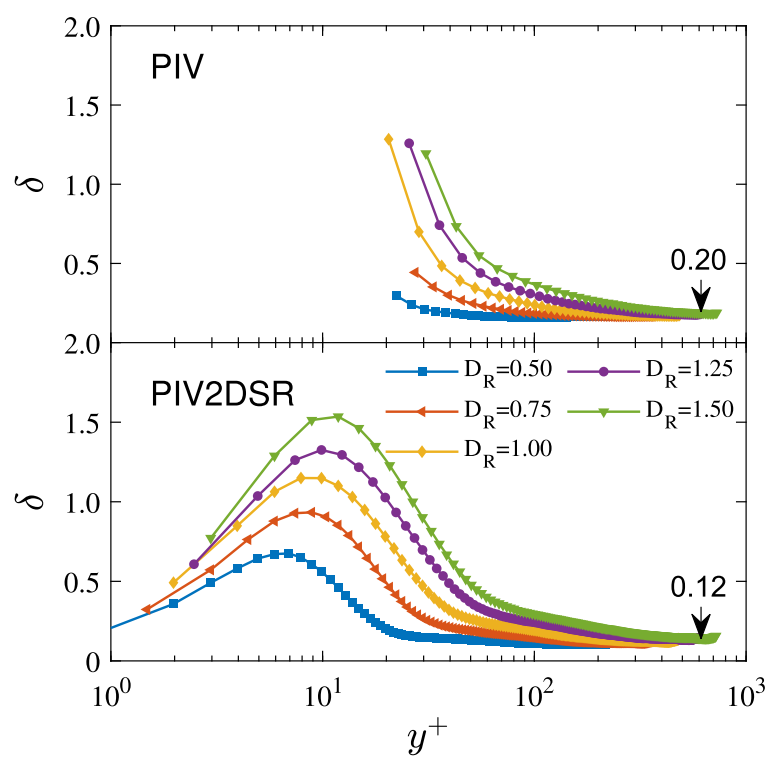

(b)

FIG. 6. Error comparison as a function of the wall-normal position $y^{+}$. Panel (a) shows the effect of the IW size $\Delta W$ on the velocity error for $p p p=0.05$ and $D_{R}=1$. Panel (b) shows the effect of the digital imaging resolution $D_{R}$ on the velocity error for $p p p=0.05$ and $\Delta W=32$.

presented in Sec. IV to prove that the proposed network can be successfully applied to improve the spatial resolution of the velocity fields obtained through PIV. Second, the performance of the network depends on the spatial resolution of the input velocity fields. More information is helpful to improve the network performance.
TABLE II. Mean errors across the boundary layer with different $\Delta W, D_{R}$, and ppp values. The errors of both PIV $\left(\delta_{P I V}\right)$ and PIV2DSR $\left(\delta_{P I V 2 D S R}\right)$ were calculated over all PIV fields above $y^{+}=D^{+}$.

\begin{tabular}{lllll}
\hline \hline$\Delta W$ & $D_{R}$ & $p P p$ & $\delta_{P I V}$ & $\delta_{P I V 2 D S R}$ \\
\hline 16 & 0.5 & 0.01 & 0.199 & 0.141 \\
16 & 0.5 & 0.05 & 0.189 & 0.131 \\
16 & 0.5 & 0.09 & 0.189 & 0.131 \\
32 & 1.0 & 0.01 & 0.242 & 0.189 \\
32 & 1.0 & 0.05 & 0.229 & 0.169 \\
32 & 1.0 & 0.09 & 0.230 & 0.169 \\
48 & 1.5 & 0.01 & 0.353 & 0.295 \\
48 & 1.5 & 0.05 & 0.343 & 0.275 \\
58 & 1.5 & 0.09 & 0.343 & 0.273 \\
\hline \hline
\end{tabular}

\section{Velocity statistics}

In this subsection, the velocity statistics obtained via PIV and PIV2DSR are compared with the DNS results, as given in Fig. 8. The left panels show the mean streamwise velocity profiles and the Reynolds stresses as functions of $y^{+}$, and the right panels present the absolute errors estimated from the values of the left panels. We will first inspect the mean velocity profiles shown in Figs. 8(a) and 8(b). The three colored lines represent the three different cases of $(\Delta W$, $\left.D_{R}\right)=(16,0.5),\left(\Delta W, D_{R}\right)=(32,1.0)$, and $\left(\Delta W, D_{R}\right)=(48,1.5)$, which are indicated in Fig. 7 (c) by red dots. For reference, the gray solid lines in Fig. 8(a) show the DNS results. The PIV profiles show good agreement with the reference lines because we consider only the velocity fields at $y^{+} \geq 20$. Smaller IWs not only allow vectors to be located closer to the wall but also result in a smaller bias because of the reduced velocity difference across the window height (Atkinson et al., 2011). In Fig. 8(b), the absolute error increases to a large value near the wall, and this behavior is consistent with the trend of the error in Fig. 6. Surprisingly, the mean velocity profiles output by the PIV2DSR model show good agreement with the DNS results even in the near-wall region where no PIV data are available. However, slight deviations from the blue and orange profiles can be observed near the wall, especially, in the region of $y^{+} \approx 10$, as shown in Fig. 8(b). Hence, the performance of PIV2DSR in this region still needs further improvement. In general, Fig. 8(a) indicates that PIV2DSR can perfectly predict the mean profile.

Now, the performance of PIV2DSR in predicting the Reynolds stresses is further evaluated. The profiles and absolute errors of Reynolds stresses $\left\langle u^{\prime 2}\right\rangle^{+},\left\langle v^{\prime 2}\right\rangle^{+}$, and $-\left\langle u^{\prime} v^{\prime}\right\rangle^{+}$are presented as functions of $y^{+}$in Figs. 8(c)-8(h), respectively. Notably, due to the insufficient number of PIV samples (500 PIV fields), the Reynolds shear stress $-\left\langle u^{\prime} v^{\prime}\right\rangle^{+}$obtained through PIV exhibits a deviation from the DNS profile, especially, in the outer layer. However, this limitation does not affect the results of the comparison. The PIV results exhibit large errors close to the wall, whereas the results of PIV2DSR show better agreement with the DNS results. Among all three colored curves, the blue and red curves are closer to the reference than the case of $\left(\Delta W, D_{R}\right)=(48,1.5)$, which shows the underestimation of the Reynolds stresses. 


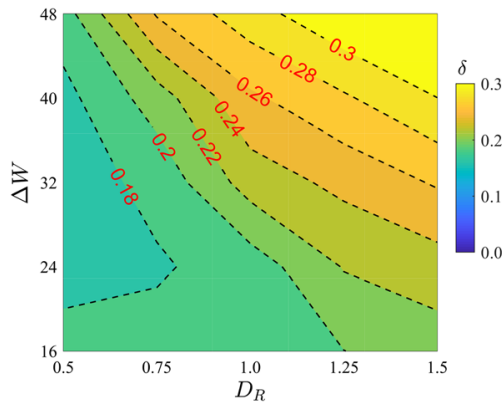

(a)

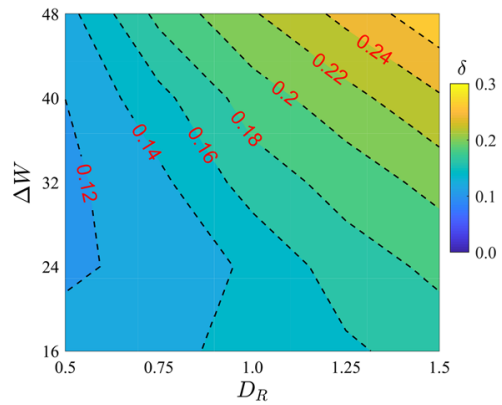

(b)

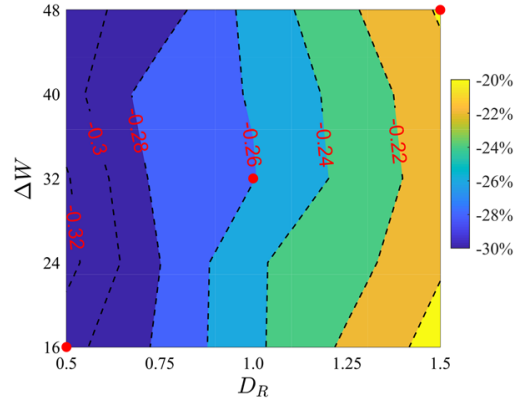

(c)

FIG. 7. Mean error across the boundary layer as a function of $\Delta W$ and $D_{R}$ at $p p p=0.05$. Panel (a) shows the error contour map of $\delta_{P I V}$, panel (b) shows the error contour map of $\delta_{P I V 2 D S R}$, and panel (c) shows the relative error reduction, estimated as $\left(\delta_{P I V 2 D S R}-\delta_{P I V}\right) / \delta_{P I V}$.

\section{Energy spectra}

The one-dimensional streamwise velocity power spectral densities (PSDs) are compared among DNS, PIV, and PIV2DSR in Fig. 9. A Hanning-windowed fast Fourier transform (FFT) was adopted to calculate the power spectra from the velocity fields to avoid frequency leakage. In Fig. 9, the spectra are plotted as a function of $k_{x} y^{+}$, where $k_{x}$ is the streamwise wavenumber. From top to bottom, the wall-normal positions used for spectral estimation are $y^{+}=200$, 50 , and 5, respectively, and from left to right, the values of $\Delta W$ and $D_{R}$ are $(16,0.5),(32,1)$, and $(48,1.5)$, respectively. The $p p p$ value is 0.05 . All PIV velocities were calculated with a fixed overlap of 75\%. According to the results given by Foucaut et al. (2004), the spatial response of PIV can be described in terms of three parameters, $k_{\min }, k_{c}$, and $k_{\max }$. The parameter $k_{\min }$ represents the lowest resolvable wavenumber corresponding to the size of the measurement domain. In the present work, $k_{\min }$ is calculated as $k_{\min }=2 \pi / L_{x}$, where $L_{x}$ is the streamwise length of the PIV domain. The cutoff wavenumber $k_{c}$ is estimated as $2.8 / \Delta W$ in accordance with the energy decrease of $-3 \mathrm{~dB}$. However, the noise in PIV often causes the effective cut-off wavenumber, denoted by $k_{\max }$, to be smaller than $k_{c}$ (Foucaut et al., 2004 and Atkinson et al., 2011). The parameter $k_{\max }$ is defined as the wavenumber at which the PIV velocity power spectrum is twice as great (or half as small) as the original DNS spectrum (Atkinson et al., 2013). Notably, the wavenumber $k_{\text {max }}$ will increase with increasing $\Delta W$ up to $k_{\max }=k_{c}$ due to the decreasing influence of PIV noise (Foucaut et al., 2004). Therefore, in the present work, we consider only the wavenumbers $k_{\min }$ and $k_{\max }$. The effective spatial dynamic range, defined as $S D R=k_{\max } / k_{\min }$, is used to evaluate the performance of PIV2DSR.

As seen in Fig. 9, in general, the spectra of PIV2DSR are closer to the DNS results than the PIV spectra are. Especially, at $y^{+}=5$, where the flow motion cannot be resolved by PIV, the PIV2DSR spectra still show good agreement with the DNS spectra in a certain wavenumber range. To present a clearer comparison, we take Figs. 9(d), 9(f), and 9(h) as examples. First, for Fig. 9(d), the value of $k_{\max }$ for PIV2DSR is 0.1818 , which is slightly larger than the corresponding value of 0.1676 for PIV. According to the definition of SDR, the SDR values for PIV2DSR and PIV are 29.3 and 27.0 , respectively. Although there is little difference in terms of $S D R$, the peel-off of the PIV spectra appearing above $k_{\max }$ is reduced, resulting in spectra that more closely follow the reference DNS spectra. This implies that the errors at high wavenumbers are smoothed by PIV2DSR without influencing the flow fields at low wavenumbers. Second, for Fig. 9(f), the SDR value for PIV2DSR is 17.8, as computed from $k_{\min }=0.0021$ and $k_{\max }=0.0373$, which is approximately twice as large as the PIV result of $S D R=8.7$. In contrast to Fig. 9(d), the error in Fig. 9(f) is dominated by the modulation effect due to the larger IW. Thus, the energy spectra are lower than the reference DNS spectra even in the range of low wavenumbers. The energy spectra of PIV2DSR show perfect agreement with the DNS spectra at low wavenumbers; however, there is still a large deviation above $k_{\max }=0.0373$. The small-scale structures lost as a result of the large IW are insufficiently recovered by the proposed network. Finally, for Fig. 9(h), the PIV spectra cannot be computed because there are no PIV data at $y^{+}=5$. Surprisingly, however, the spectra of PIV2DSR still show good agreement with the DNS spectra, especially, in the range of $k_{x} \in[0.0031,0.0785]$. These findings indicate that the large-scale structures at $y^{+}=5$ can be effectively predicted by the neural network.

\section{EXPERIMENTAL MEASUREMENTS OF A TURBULENT BOUNDARY LAYER}

\section{A. Experimental setup}

Experimental measurements of a turbulent boundary layer were conducted in a water tunnel at Beihang University, China [Wang et al., 2018; 2019a; and 2019b]. The dimensions of the main test section of the water tunnel are $\sim 18 \times 1.2 \times 1 \mathrm{~m}^{3}$ (length $\times$ height $\times$ width). The turbulence level is $\sim 0.8 \%$ at a typical freestream velocity $U_{\infty}$ of $0.5 \mathrm{~m} / \mathrm{s}$. Figure 10 shows the setup for this experiment. To generate a turbulent boundary layer, a smooth and flat acrylic glass plate with dimensions of $15 \times 1 \mathrm{~m}^{2}$ (length $\times$ width) was vertically mounted in the tunnel, $\sim 0.75 \mathrm{~m}$ from the sidewall. Its leading edge was processed to an elliptical shape. At $0.4 \mathrm{~m}$ downstream of the leading edge, a trip wire with a diameter of $5 \mathrm{~mm}$ was glued to the wall to accelerate the turbulence transition. The measurement domain was located $12 \mathrm{~m}$ from the leading edge and at the center of the tunnel in the spanwise direction. A laser sheet of $1 \mathrm{~mm}$ in thickness generated by a double-pulsed high-power laser (Beamtech Vlite-500) was used to illuminate the tracer particles. The laser plane 


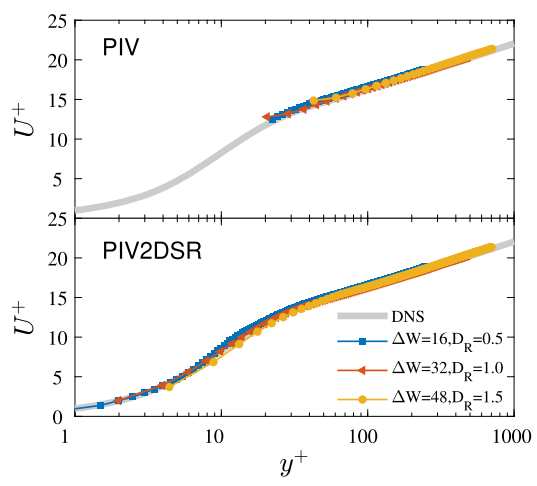

(a)

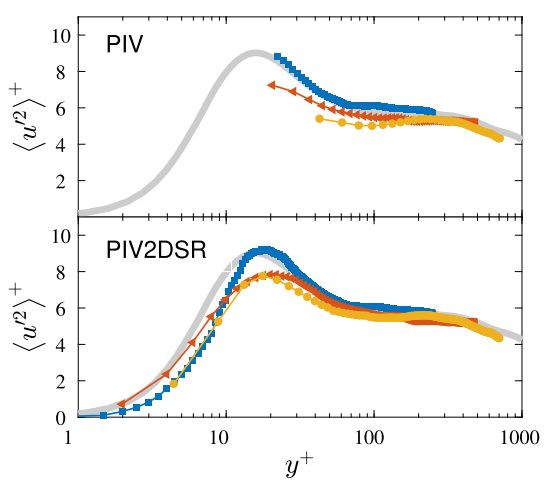

(c)

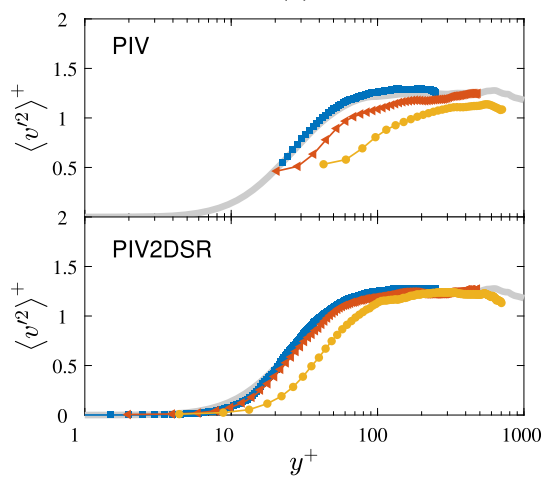

(e)

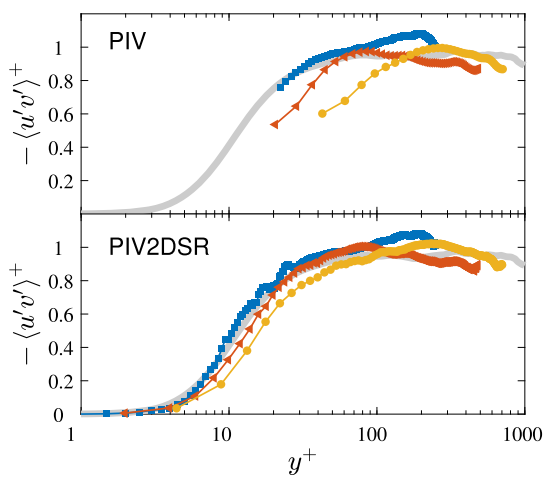

(g)

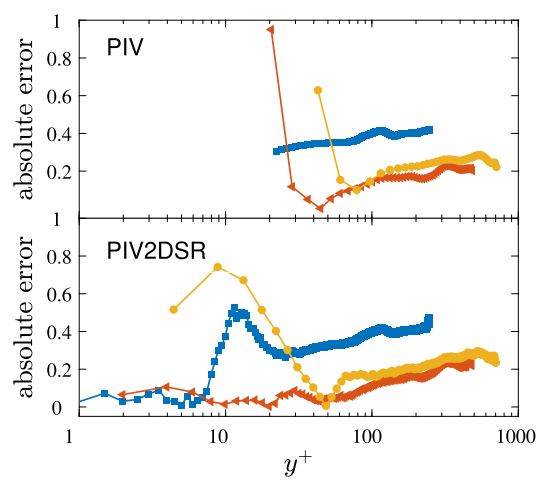

(b)

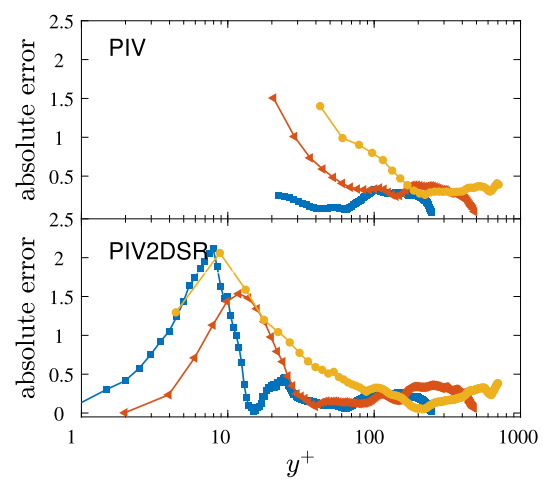

(d)

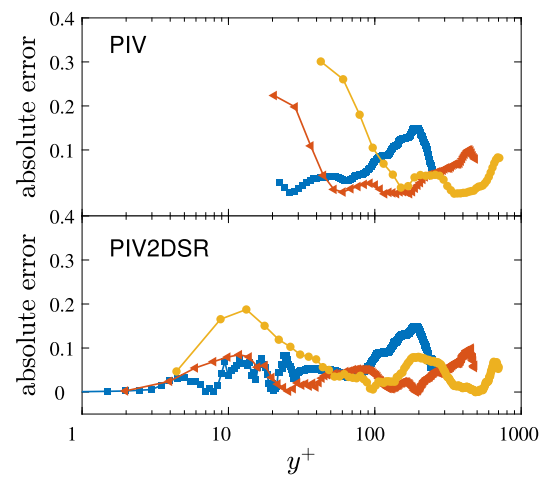

(f)

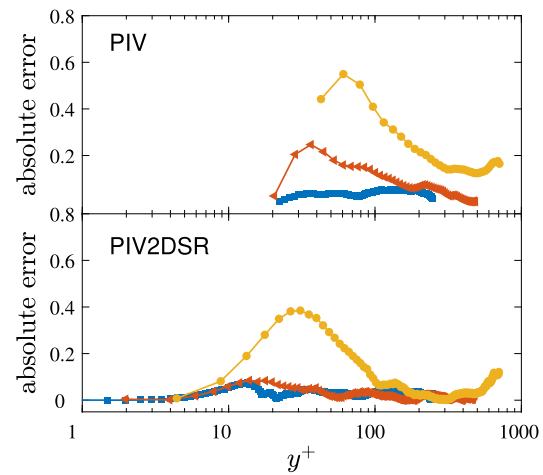

(h)
FIG. 8. Left panels: Comparison of the mean streamwise velocity profiles and the Reynolds stresses as functions of $y^{+}$. Right panels: Absolute errors of PIV and PIV2DSR compared with the DNS. Panels (a) and (b) show the mean velocity profiles, panels (c) and (d) show the Reynolds stress $\left\langle u^{\prime 2}\right\rangle^{+}$, panels (e) and (f) show the Reynolds stress $\left\langle v^{\prime 2}\right\rangle^{+}$, and panels $(\mathrm{g})$ and $(\mathrm{h})$ show the Reynolds stress $-\left\langle u^{\prime} v^{\prime}\right\rangle^{+}$. 


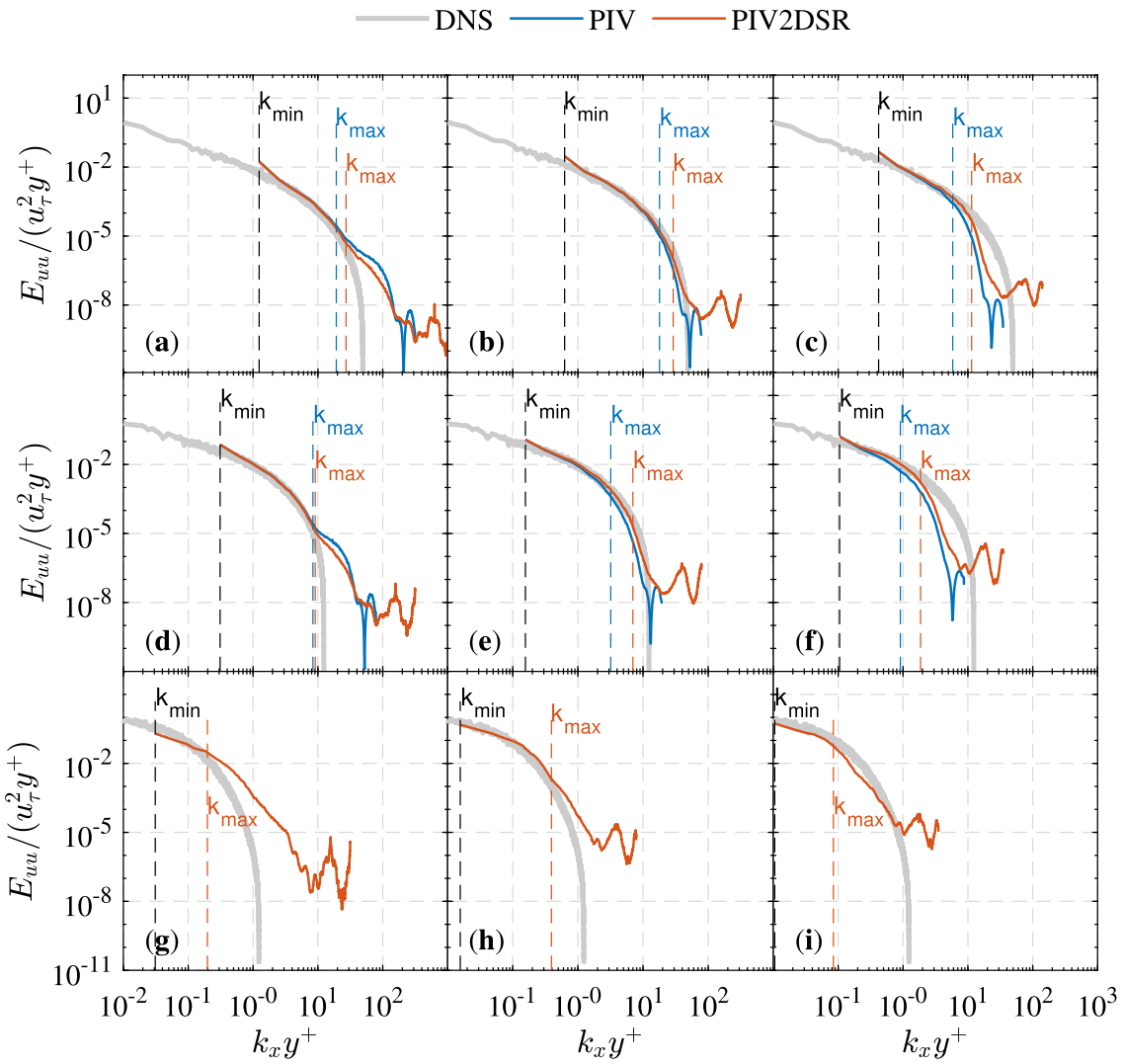

FIG. 9. One-dimensional streamwise velocity PSDs for DNS (gray), PIV (blue), and PIV2DSR (red). From top to bottom, the $y^{+}$positions are $200[(\mathrm{a})-(\mathrm{c})], 50$ $[(d)-(f)]$, and $5[(g)-(i)]$. From left to right, the $\left(\Delta W, D_{R}\right)$ configurations are $(16$, $0.5)[(a),(d)$, and $(g)],(32,1)[(b),(e)$, and $(\mathrm{h})]$, and $(48,1.5)[(\mathrm{c}),(\mathrm{f})$, and (i)]. There are no PIV data for panels $(\mathrm{g}),(\mathrm{h})$ and (i).

was perpendicular to the wall (acrylic glass plate) and parallel to the streamwise-wall-normal plane. We again use $x$ and $y$ to denote the streamwise and wall-normal directions, respectively, and the corresponding velocity components are denoted by $u$ and $v$, respectively. A 12-bit CCD camera (IMPERX B2520) with a $50 \mathrm{~mm}$ Nikon camera lens was used to record particle images at a repetition rate of $2 \mathrm{~Hz}$, with an imaging size of $2456 \times 2058 \mathrm{pixel}^{2}$. The FOV was $\sim 108$ $\times 140 \mathrm{~mm}^{2}$ corresponding to a digital resolution of $0.057 \mathrm{~mm} /$ pixel and began at the wall.

The flow field was calibrated using 1D laser Doppler velocimetry (LDV) measurements. The mean velocity profile was fitted to estimate the properties of the turbulent boundary layer, which are given in Table III, using the Musker profile method proposed by

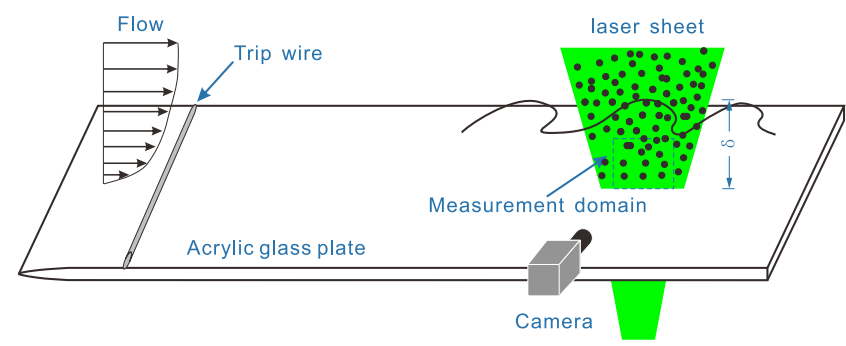

FIG. 10. Schematic of the experimental setup.
Kendall and Koochesfahani (2008). The friction Reynolds number $R e_{\tau}$ was 2200 , corresponding to the boundary thickness of $\delta=0.199 \mathrm{~m}$, the inner length scale of $y^{*}=0.0869 \mathrm{~mm}$, and the friction velocity of $u_{\tau}=0.295 \mathrm{~m} / \mathrm{s}$. The FOV expressed in WUs was 1242 $\times 1611$, and the digital imaging resolution $D_{R}$ was $\sim 0.656 \mathrm{WUs} /$ pixel.

The $2 \mathrm{D}$ velocity field was also estimated using a three-pass WIDIM. Three different IW sizes of $64 \times 8$ pixels $^{2}, 32 \times 32$ pixels $^{2}$, and $48 \times 48$ pixels $^{2}$ were adopted to estimate the velocity field with vector spacings of 4 pixels, 8 pixels, and 12 pixels, respectively, in both the $x$ and $y$ directions. For convenience, these three cases are denoted by PIV8, PIV32, and PIV48, respectively. A rectangle IW in PIV8 is applied to increase the wall-normal resolution, and the highest-resolution field is used as a visual reference for evaluating the velocity fields obtained using PIV2DSR from PIV32 and PIV48. The velocity fields are smoothed by a Gaussian filter with a kernel size of $\left(\Delta_{x}, \Delta_{y}\right)=(7,3)$ and a standard deviation of $\left(\sigma_{x}, \sigma_{y}\right)=\left(\Delta_{x} / 4, \Delta_{y} / 4\right)$. The statistical analysis is performed over 700

TABLE III. Properties of the turbulent boundary layer under investigation.

\begin{tabular}{ll}
\hline \hline Freestream velocity & $U_{\infty}=0.295(\mathrm{~m} / \mathrm{s})$ \\
Reynolds number & $R e_{\tau}=2200$ \\
Friction velocity & $u_{\tau}=0.0118 \mathrm{~m} / \mathrm{s}$ \\
Boundary layer thickness & $\delta=0.199 \mathrm{~m}$ \\
Inner length scale & $y^{*}=0.0869 \mathrm{~mm}$ \\
\hline
\end{tabular}


velocity fields. Meanwhile, the statistical data extracted from DNS results for a fully developed zero-pressure-gradient (ZPG) boundary layer flow (Simens et al., 2009 and Sillero et al., 2013) and a turbulent channel flow (Lee and Moser, 2015) at $R e_{\tau}=2000$ are also considered for comparison with the experimental data. All velocity statistics are available from https://torroja.dmt.upm.es/turbdata/blayers/ and https://turbulence.oden.utexas.edu/. In the present work, the boundary layer flow and channel flow data are denoted by B2000 and C2000, respectively. The experimental velocity fields are analyzed in terms of the velocity statistics and streamwise velocity energy spectra.

\section{B. Velocity statistics}

Instantaneous fields of streamwise velocity and vorticity are visualized in Figs. 11(a) and 11(b), respectively. From left to right, the results of PIV32, PIV2DSR, and PIV8 are presented for comparison. Due to the insufficient number of tracer particles in the small IW, the velocity field of PIV8 contains more random noise and clustered outliers that are difficult to detect and remove (Shinneeb et al., 2004 and Wang et al., 2015). The first near-wall points of the velocity field are at $y^{+}=26.4$ and 2.6 for PIV32 and PIV8, respectively, whereas the first near-wall location for the high-resolution field obtained by PIV2DSR is $y^{+}=1.3$. In the outer layer, the velocity fields of PIV32 and PIV2DSR are identical to that of PIV8, implying that the resolution of PIV32 is sufficient to resolve the small-scale flow structures. This conclusion is also supported by the energy spectrum analysis in Sec. IV C. The velocity field in the near-wall region

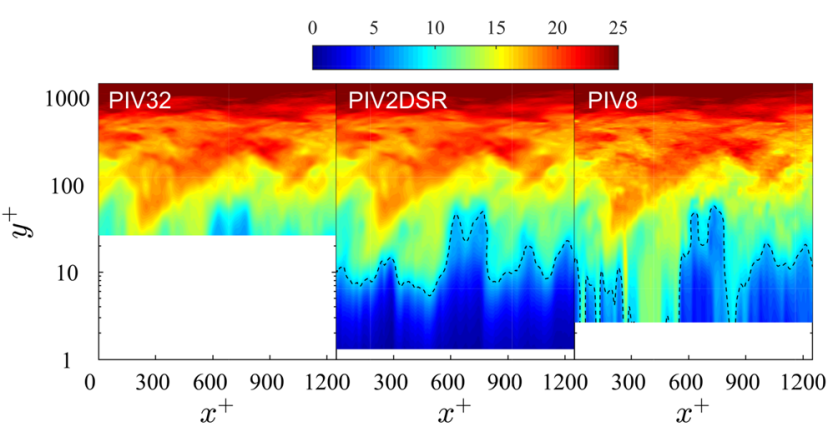

(a)

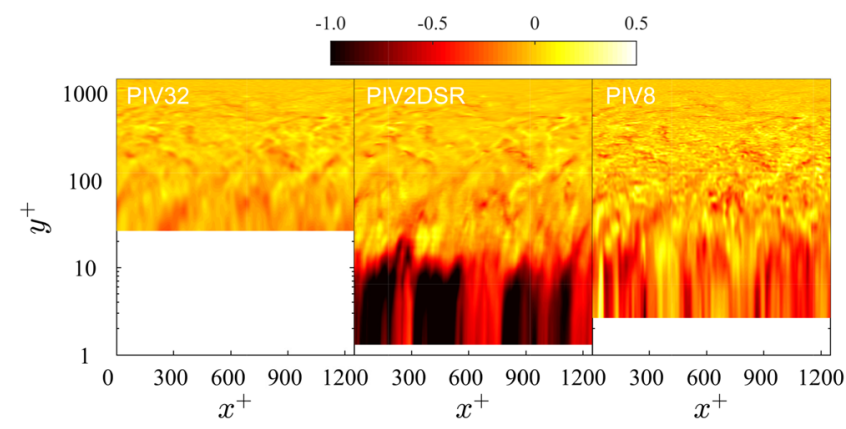

(b)

FIG. 11. Instantaneous fields of streamwise velocity (a) and vorticity (b). can be predicted by PIV2DSR. As seen in Fig. 11(a), the trend of the contour line of $u^{+}=8$ for PIV2DSR is close to the PIV8 result, indicating that the PIV2DSR model can be successfully applied to experimental PIV fields.

The mean velocity profiles measured in the PIV experiment and predicted by PIV2DSR as well as the Reynolds stresses are presented in Fig. 12. The results of PIV32 and PIV48 are presented on the left and right, respectively. For comparison, the profiles of the boundary layer and channel flows at $R e_{\tau}=2000$ are also given in Fig. 12 . The velocity statistics for the channel and boundary layer flows show perfect agreement in the inner layer of $y^{+}<50$. The channel flow velocity statistics are presented in this figure because the PIV2DSR model was trained entirely on channel flow data. With increasing IW from 32 pixels to 48 pixels, the velocity fluctuations of PIV are significantly underestimated, as shown in Fig. 12. For the mean velocity profile and Reynolds stresses $\left\langle u^{\prime 2}\right\rangle^{+}$and $\left\langle v^{\prime 2}\right\rangle^{+}$, the red predicted solid curves show acceptable agreement with the DNS results. However, for Reynolds shear stress $-\left\langle u^{\prime} v^{\prime}\right\rangle^{+}$, the red curve obtained via PIV2DSR exhibits an incorrect peak. The potential reasons for the large deviation on Reynolds shear stress prediction will be discussed in Sec. IV D.

\section{Energy spectra}

The energy spectra of PIV32, PIV2DSR, and the turbulent channel flow are also compared in Fig. 13. From left to right, the wall-normal locations $y^{+}$are 100,30 , and 5. There are no PIV data at $y^{+}=5$. The spectra of PIV32 at $y^{+}=100$ and 30 show good agreement with the DNS spectra even at the largest $k_{x}$, implying that the resolution of PIV32 is sufficiently high to resolve the turbulence, as discussed in Sec. IV B. The energy spectra of PIV2DSR exhibit little difference from those of PIV32 at $y^{+}=100$ and 30. However, the prediction at $y^{+}=5$ shows overestimation with respect to the DNS result. From the energy spectra, it seems that PIV2DSR encounters nonphysical noise at high wavenumbers.

\section{Discussion}

From the real experimental results, we found that the prediction performance of PIV2DSR on experimental PIV fields is worse than that of synthetic PIV fields. Especially, for high-order statistics, the prediction of PIV2DSR presents a large deviation near the wall for the experimental data. In our opinion, there are three possible reasons. First, the random errors caused by the image noise, variation of particle intensity, a reduced seeding concentration, local particle image distortions, and out-of-plane motions (Atkinson et al., 2013 and Wang et al., 2015) are not considered in this network model. Consequently, such errors may reduce the performance of PIV2DSR. To investigate the influence of random error in real experiments, the velocity fields of PIV32 were smoothed by a Gaussian filter with a kernel size of $\left(\Delta_{x}, \Delta_{y}\right)$ and a standard deviation of $\left(\sigma_{x}, \sigma_{y}\right)=\left(\Delta_{x} / 4, \Delta_{y} / 4\right)$. The different values of the streamwise kernel size $\Delta_{x}$ are 7,15 , and 23 , and the wall-normal kernel size $\Delta_{y}$ is fixed to 3. Large $\Delta_{x}$ means smoother velocity fields. The predicted $-\left\langle u^{\prime} v^{\prime}\right\rangle^{+}$ profiles from the different filtered fields are presented in Fig. 14. The predicted profiles gradually move toward the DNS results with increasing $\Delta_{x}$. Meanwhile, the prediction of $-\left\langle u^{\prime} v^{\prime}\right\rangle^{+}$for PIV48 is also better than that of PIV32, as shown in Fig. 12(h). Therefore, we 


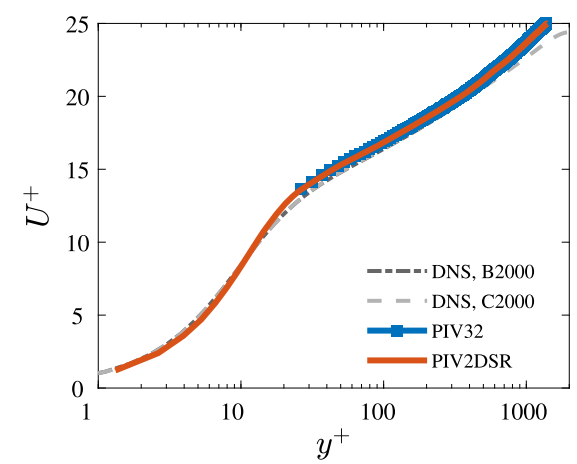

(a)

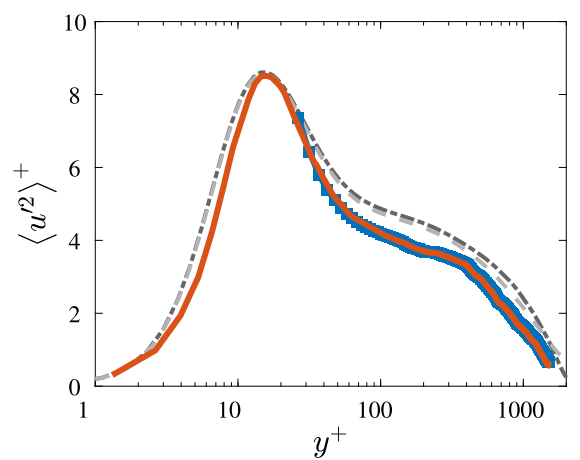

(c)

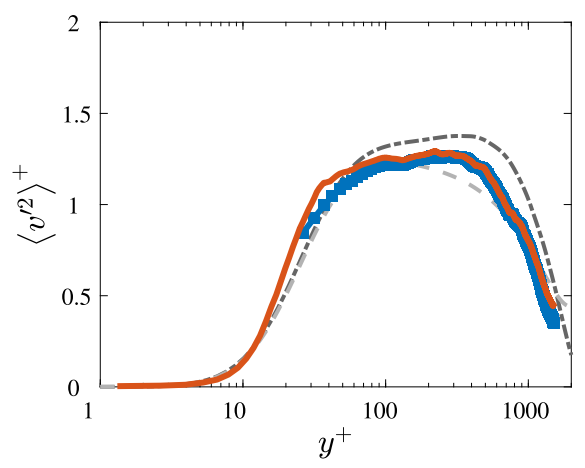

(e)

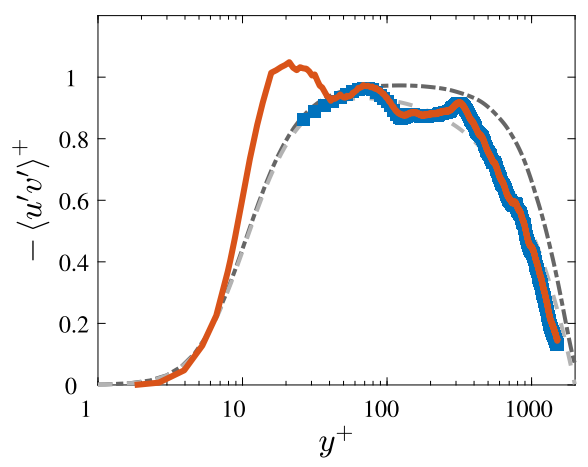

(g)

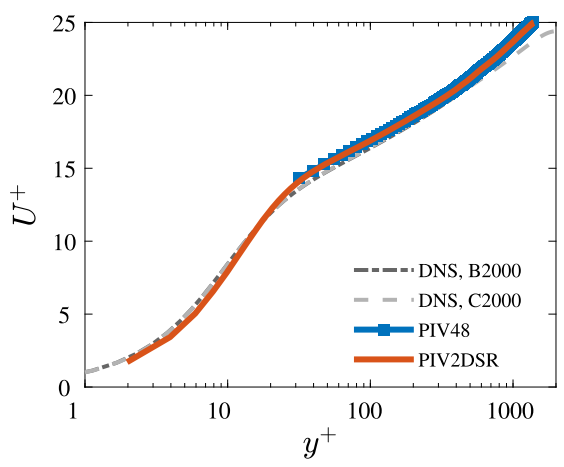

(b)

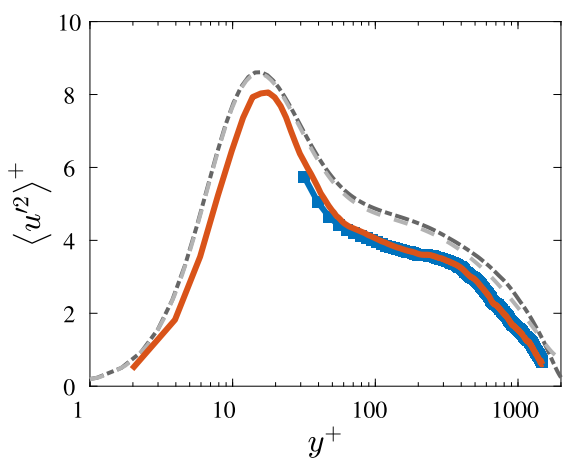

(d)

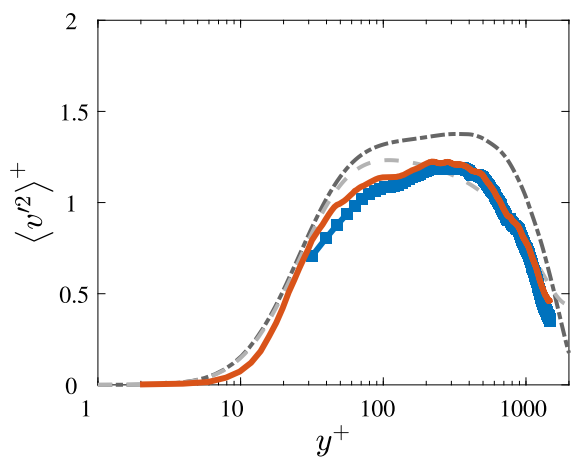

(f)

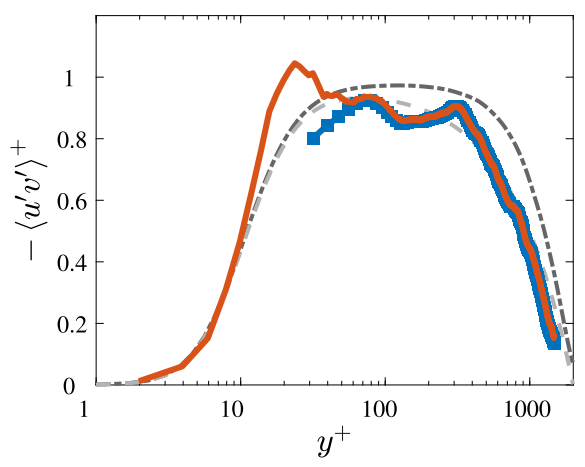

(h)
FIG. 12. Comparison of the mean streamwise velocity profiles and Reynolds stresses as functions of $y^{+}$. Panels (a) and (b) show the mean velocity profiles, panels (c) and (d) show the Reynolds stress $\left\langle u^{\prime 2}\right\rangle^{+}$, panels (e) and (f) show the Reynolds stress $\left\langle v^{\prime 2}\right\rangle^{+}$, and panels $(\mathrm{g})$ and $(\mathrm{h})$ show the Reynolds stress $-\left\langle u^{\prime} v^{\prime}\right\rangle^{+}$. Panels (a), (c), (e), and (g) show the results of PIV32, and panels (b), (d), (f), and (h) show the results of PIV48. 


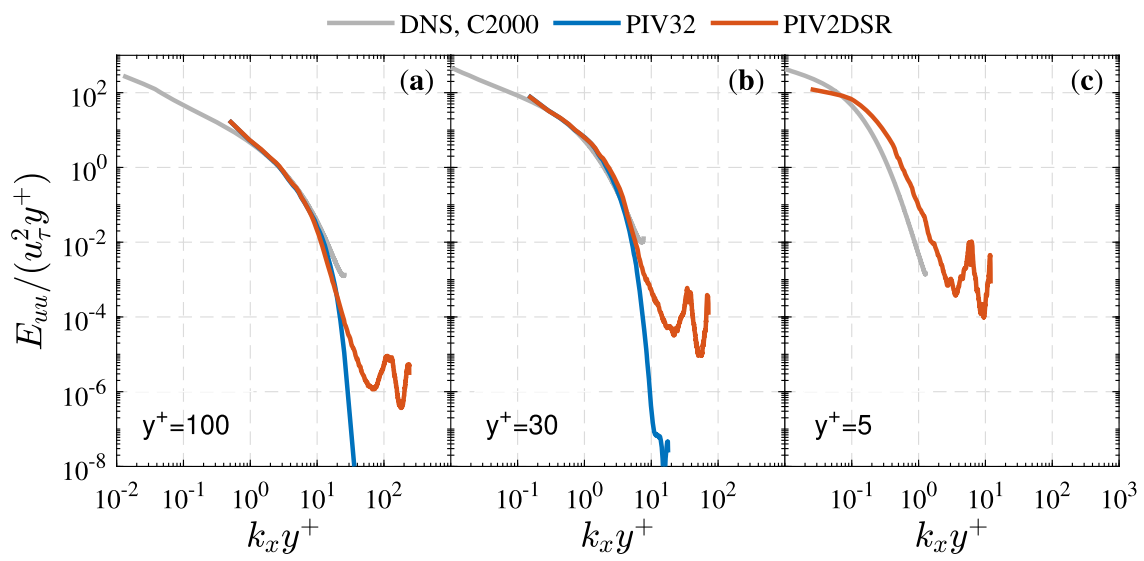

FIG. 13. One-dimensional streamwise velocity PSDs for DNS (gray), PIV32 (blue), and PIV2DSR (red). From left to right, the $y^{+}$positions are $100(a), 30(b)$ and 5 (c). There are no PIV data for pane (c).

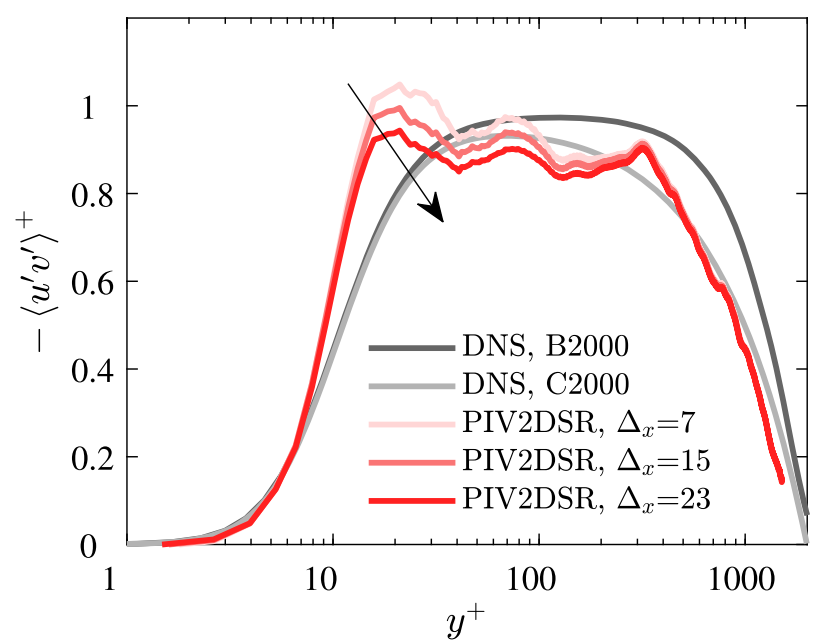

FIG. 14. Predicted Reynolds shear stress profiles at three different streamwise filter sizes $\Delta_{x}$ for PIV32. The wall-normal filter size $\Delta_{y}$ remains a constant of 3.

believe that the random error in real PIV experiments is the dominant reason for the large deviation. Second, the PIV2DSR model was trained only on turbulent channel flow data. The velocity statistics of the turbulent boundary layer present good agreement with that of the turbulent channel flow at $y^{+}<50$. The universal near-wall structures presented in wall-bounded turbulence make the prediction possible. However, the statistical characteristics of these two kinds of wall turbulence are different in the outer layer. This may reduce the performance of the network. Third, the loss function only considers the MSE between the output velocity and ground-truth velocity during training. A potential way to reduce the large deviation of Reynolds shear stress is to consider the $-\left\langle u^{\prime} v^{\prime}\right\rangle^{+}$fields in the loss function.

\section{CONCLUSIONS}

This study presents a machine-learning-based approach for predicting the near-wall velocity of PIV fields in cases of wall-bounded turbulence. The proposed network, named PIV2DSR, is constructed using convolutional layers and residual blocks and can upscale the resolution of input velocity fields by a factor of 4 . To train the network, a large number of synthetic PIV fields were generated from DNS velocity fields of channel flows at $R e_{\tau}=1000$. The PIV vectors in the region of $y^{+} \leq 20$ were replaced through linear extrapolation. These PIV fields served as the input to the PIV2DSR model. The corresponding ground truth was generated by spline interpolating the reference DNS data. The weights of PIV2DSR were optimized by minimizing the MSE between the output and ground-truth fields. After establishing the relationship between the low-resolution and high-resolution fields, the trained PIV2DSR model was used to predict the instantaneous fields near the wall.

A test dataset of channel flows at $R e_{\tau}=5200$ was generated to evaluate the performance of PIV2DSR. The influences of the particle concentration $p p p$, the digital imaging resolution $D_{R}$, and the IW size $\Delta W$ were investigated when generating the test set. The performance of PIV2DSR was analyzed in terms of instantaneous fields, error analysis, velocity statistics, and energy spectra. Three conclusions can be drawn from the results. First, PIV2DSR has a good generalization ability among various experimental conditions ( $p$ p , $D_{R}$, and $\left.\Delta W\right)$. Second, the high-resolution fields recovered from corresponding low-resolution fields show good agreement with the ground-truth fields in terms of the Reynolds stresses and energy spectra, and the error of the PIV fields is also reduced. The effective spatial dynamic range $S D R$ achieved with PIV2DSR is increased by approximately a factor of 2 at $y^{+}=50$. Third, the predicted velocity fields near the wall are still acceptable. The instantaneous fields, velocity statistics, and large-scale spectra are quite consistent with those of the reference data.

The performance of PIV2DSR was further investigated based on real experimental PIV data for a turbulent boundary layer. The mean velocity $U^{+}$and Reynolds stresses $\left\langle u^{\prime 2}\right\rangle^{+}$and $\left\langle v^{\prime 2}\right\rangle^{+}$predicted by PIV2DSR show good agreement with the reference DNS profiles, while the profiles of $-\left\langle u^{\prime} v^{\prime}\right\rangle^{+}$exhibit large deviations. As discussed in Sec. IV D, this is mainly because the real PIV data contain random error, which is not considered in the model. To address these problems, we may consider introducing a more restrictive loss function or physical constraints into the neural network in future work. 
We believe that this machine-learning-based technique can be used to perform outlier detection, velocity correction, and resolution enhancement for PIV data and can even be used to predict missing PIV data where the flow motion cannot be evaluated by means of PIV. Moreover, this approach can also be used in numerical simulations to develop subgrid models or near-wall turbulence models.

\section{ACKNOWLEDGMENTS}

This work was supported by the NSFC Basic Science Center Program for "Multiscale Problems in Nonlinear Mechanics" (Grant No. 11988102) and by the National Natural Science Foundation of China (Grant Nos. 11702302, 11922214, and 91752118). The authors would also like to acknowledge the support from the Strategic Priority Research Program (Grant No. XDB22040104) and the Key Research Program of Frontier Sciences of the Chinese Academy of Sciences (Grant No. QYZDJ-SSW-SYS002).

\section{DATA AVAILABILITY}

The data that support the findings of this study are available from the corresponding author upon reasonable request.

\section{REFERENCES}

Abbassi, M. R., Baars, W. J., Hutchins, N., and Marusic, I., "Skin-friction drag reduction in a high-Reynolds-number turbulent boundary layer via real-time control of large-scale structures," Int. J. Heat Fluid Flow 67, 30-41 (2017).

Adrian, R. J., “Twenty years of particle image velocimetry," Exp. Fluids 39, 159169 (2005).

Atkinson, C., Buchmann, N., Amili, O., and Soria, J., "On the appropriate filtering of PIV measurements of turbulent shear flows,” Exp. Fluids 55, 1654 (2013).

Atkinson, C., Coudert, S., Foucaut, J.-M., Stanislas, M., and Soria, J., “The accuracy of tomographic particle image velocimetry for measurements of a turbulent boundary layer," Exp. Fluids 50, 1031-1056 (2011).

Brunton, S. L., Noack, B. R., and Koumoutsakos, P., "Machine learning for fluid mechanics," Annu. Rev. Fluid Mech. 52, 477 (2020).

Christensen, K. T. and Adrian, R. J., "Statistical evidence of hairpin vortex packets in wall turbulence," J. Fluid Mech. 431, 433-443 (2001).

Deng, S., Pan, C., Wang, J., and He, G., "On the spatial organization of hairpin packets in a turbulent boundary layer at low-to-moderate Reynolds number," J. Fluid Mech. 844, 635-668 (2018)

Deng, Z., He, C., Liu, Y., and Kim, K., "Super-resolution reconstruction of turbulent velocity fields using a generative adversarial network-based artificial intelligence framework," Phys. Fluids 31, 125111 (2019).

de Giovanetti, M., Hwang, Y., and Choi, H., "Skin-friction generation by attached eddies in turbulent channel flow," J. Fluid Mech. 808, 511-538 (2016).

Dong, C., Loy, C. C., He, K., and Tang, X., "Learning a deep convolutional network for image super-resolution," in Computer Vision-ECCV 2014, Pt Iv, Lecture Notes in Computer Science, Vol. 8692, edited by Fleet, D., Pajdla, T., Schiele, B., and Tuytelaars, T. (Springer International Publishing Ag, Cham, 2014), pp. 184-199.

Duraisamy, K., Iaccarino, G., and Xiao, H., "Turbulence modeling in the age of data," Annu. Rev. Fluid Mech. 51, 357-377 (2019).

Foucaut, J. M., Carlier, J., and Stanislas, M., "PIV optimization for the study of turbulent flow using spectral analysis," Meas. Sci. Technol. 15, 1046-1058 (2004).

Fukami, K., Fukagata, K., and Taira, K., "Super-resolution reconstruction of turbulent flows with machine learning," J. Fluid Mech. 870, 106-120 (2019).
Glorot, X., Bordes, A., and Bengio, Y., "Deep sparse rectifier neural networks," in Proceedings of the Fourteenth International Conference on Artificial Intelligence and Statistics (JMLR, Cambridge, MA, 2011), pp. 315-323.

Graham, J., Kanov, K., Yang, X. I. A., Lee, M., Malaya, N., Lalescu, C. C., Burns, R., Eyink, G., Szalay, A., Moser, R. D., and Meneveau, C., "A web services accessible database of turbulent channel flow and its use for testing a new integral wall model for LES," J. Turbul. 17, 181-215 (2016).

Hutchins, N. and Marusic, I., "Evidence of very long meandering features in the logarithmic region of turbulent boundary layers," J. Fluid Mech. 579, 1-28 (2007).

Jiménez, J. and Pinelli, A., “The autonomous cycle of near-wall turbulence," J. Fluid Mech. 389, 335-359 (1999).

Karimpour, F. and Venayagamoorthy, S. K., "Some insights for the prediction of near-wall turbulence,” J. Fluid Mech. 723, 126-139 (2013).

Ke, Q., Liu, J., Bennamoun, M., An, S., Sohel, F., and Boussaid, F., "Chapter 5Computer vision for human-machine interactions," in Computer Vision for Assistive Healthcare, edited by Leo, M. and Farinella, G. M. (Academic Press, 2018), pp. 127-145.

Kendall, A. and Koochesfahani, M., "A method for estimating wall friction in turbulent wall-bounded flows,” Exp. Fluids 44, 773-780 (2008).

Kline, S. J., Reynolds, W. C., Schraub, F. A., and Runstadler, P. W., "The structure of turbulent boundary layers," J. Fluid Mech. 30, 741-773 (1967).

Lecordier, B. and Westerweel, J., "The EUROPIV synthetic image generator (S.I.G.)," in Particle Image Velocimetry: Recent Improvements, edited by Stanislas, M., Westerweel, J., and Kompenhans, J. (Springer Berlin Heidelberg, 2004), Sec. 11, pp. 145-161.

Ledig, C., Theis, L., Huszar, F., Caballero, J., Cunningham, A., Acosta, A., Aitken, A., Tejani, A., Totz, J., Wang, Z. H., and Shi, W. Z., "Photo-realistic single image super-resolution using a generative adversarial network," in 30th IEEE Conference on Computer Vision and Pattern Recognition (IEEE, New York, 2017), pp. 105-114.

Lee, M. and Moser, R. D., "Direct numerical simulation of turbulent channel flow up to $R e_{\tau} \approx 5200$," J. Fluid Mech. 774, 395-415 (2015).

Li, Y., Chang, J., Kong, C., and Wang, Z., "Flow field reconstruction and prediction of the supersonic cascade channel based on a symmetry neural network under complex and variable conditions," AIP Adv. 10, 065116 (2020).

Li, Y., Perlman, E., Wan, M., Yang, Y., Meneveau, C., Burns, R., Chen, S., Szalay, A., and Eyink, G., "A public turbulence database cluster and applications to study Lagrangian evolution of velocity increments in turbulence," J. Turbul. 9, N31 (2008).

Lim, B., Son, S., Kim, H., Nah, S., and Lee, K. M., "Enhanced deep residual networks for single image super-resolution," in 2017 IEEE Conference on Computer Vision and Pattern Recognition Workshops (IEEE, New York, 2017), pp. 1132-1140.

Liu, B., Tang, J., Huang, H., and Lu, X.-Y., "Deep learning methods for superresolution reconstruction of turbulent flows,” Phys. Fluids 32, 025105 (2020).

Marusic, I., Mathis, R., and Hutchins, N., "High Reynolds number effects in wall turbulence," Int. J. Heat Fluid Flow 31, 418-428 (2010a).

Marusic, I., Mathis, R., and Hutchins, N., "Predictive model for wall-bounded turbulent flow," Science 329, 193-196 (2010b).

Mathis, R., Hutchins, N., and Marusic, I., "A predictive inner-outer model for streamwise turbulence statistics in wall-bounded flows," J. Fluid Mech. 681, 537-566 (2011).

Perlman, E., Burns, R., Li, Y., and Meneveau, C., "Data exploration of turbulence simulations using a database cluster," in ACM/IEEE Conference on High Performance Networking and Computing (IEEE, Reno, Nevada, USA, 2007), pp. 1-11.

Pope, S., Turbulent Flows (Cambridge University Press, 2000).

Raffel, M., Willert, C. E., Scarano, F., Kähler, C. J., Wereley, S. T., and Kompenhans, J., Particle Image Velocimetry: A Practical Guide (Springer, 2018).

Renganathan, S. A., Maulik, R., and Rao, V., "Machine learning for nonintrusive model order reduction of the parametric inviscid transonic flow past an airfoil," Phys. Fluids 32, 047110 (2020).

Scarano, F., "Iterative image deformation methods in PIV," Meas. Sci. Technol. 13, R1-R19 (2002). 
Scarano, F., "Tomographic PIV: Principles and practice," Meas. Sci. Technol. 24, 012001 (2013).

Scarano, F. and Riethmuller, M. L., "Advances in iterative multigrid PIV image processing," Exp. Fluids 29, S051 (2000).

Schneiders, J. F. G., Scarano, F., and Elsinga, G. E., "Resolving vorticity and dissipation in a turbulent boundary layer by tomographic PTV and VIC+," Exp. Fluids 58, 27 (2017).

Schoppa, W. and Hussain, F., "Coherent structure generation in near-wall turbulence," J. Fluid Mech. 453, 57-108 (2002).

Sciacchitano, A., Wieneke, B., and Scarano, F., "PIV uncertainty quantification by image matching," Meas. Sci. Technol. 24, 045302 (2013).

Shi, W., Caballero, J., Huszàr, F., Totz, J., Aitken, A., Bishop, R., Rueckert, D., and Wang, Z., "Real-time single image and video super-resolution using an efficient sub-pixel convolutional neural network," in 2016 IEEE Conference on Computer Vision and Pattern Recognition (CVPR) (IEEE, 2016).

Shinneeb, A.-M., Bugg, J. D., and Balachandar, R., "Variable threshold outlier identification in PIV data," Meas. Sci. Technol. 15, 1722 (2004).

Sillero, J. A., Jiménez, J., and Moser, R. D., "One-point statistics for turbulent wallbounded flows at Reynolds numbers up to $\delta^{+} \approx 2000$," Phys. Fluids 25, 105102 (2013).

Simens, M. P., Jiménez, J., Hoyas, S., and Mizuno, Y., "A high-resolution code for turbulent boundary layers," J. Comput. Phys. 228, 4218-4231 (2009).

Smits, A. J., McKeon, B. J., and Marusic, I., "High-Reynolds number wall turbulence," Annu. Rev. Fluid Mech. 43, 353-375 (2011).

Stanislas, M., "Near wall turbulence: An experimental view," Phys. Rev. Fluids 2 , 100506 (2017)

Townsend, A. A., The Structure of Turbulent Shear Flow, Cambridge Monographs on Mechanics and Applied Mathematics. (Cambridge University Press, Cambridge, England; New York, 1976).

Wang, C., Gao, Q., Wang, J., Wang, B., and Pan, C., "Experimental study on dominant vortex structures in near-wall region of turbulent boundary layer based on tomographic particle image velocimetry," J. Fluid Mech. 874, 426-454 (2019a).

Wang, H. P., Gao, Q., Feng, L. H., Wei, R. J., and Wang, J. J., "Proper orthogonal decomposition based outlier correction for PIV data," Exp. Fluids 56, 43 (2015).

Wang, J., Pan, C., and Wang, J., "Characteristics of fluctuating wall-shear stress in a turbulent boundary layer at low-to-moderate Reynolds number," Phys. Rev. Fluids 5, 074605 (2020).
Wang, W., Pan, C., and Wang, J., "Multi-component variational mode decomposition and its application on wall-bounded turbulence," Exp. Fluids 60, 95 (2019b).

Wang, W., Pan, C., and Wang, J., "Quasi-bivariate variational mode decomposition as a tool of scale analysis in wall-bounded turbulence," Exp. Fluids 59, 1 (2018).

Westerweel, J., Elsinga, G. E., and Adrian, R. J., "Particle image velocimetry for complex and turbulent flows," Annu. Rev. Fluid Mech. 45, 409-436 (2013).

Westerweel, J. and Scarano, F., "Universal outlier detection for PIV data," Exp. Fluids 39, 1096-1100 (2005).

$\mathrm{Xu}, \mathrm{D}$. and Chen, J., "Accurate estimate of turbulent dissipation rate using PIV data," Exp. Therm. Fluid Sci. 44, 662-672 (2013).

Yang, X. I. A., Zafar, S., Wang, J.-X., and Xiao, H., "Predictive large-eddysimulation wall modeling via physics-informed neural networks," Phys. Rev. Fluids 4, 034602 (2019).

Yin, G., Huang, W.-X., and Xu, C.-X., "Prediction of near-wall turbulence using minimal flow unit," J. Fluid Mech. 841, 654-673 (2018).

Zaripov, D., Li, R., and Dushin, N., "Dissipation rate estimation in the turbulent boundary layer using high-speed planar particle image velocimetry," Exp. Fluids 60, 18 (2019).

Zhang, J. and Ma, W., "Data-driven discovery of governing equations for fluid dynamics based on molecular simulation," J. Fluid Mech. 892, A5 (2020).

Zhang, X., Wu, J., Coutier-Delgosha, O., and Xiao, H., "Recent progress in augmenting turbulence models with physics-informed machine learning," J. Hydrodyn. 31, 1153-1158 (2019a).

Zhang, X., Xiao, H., Gomez, T., and Coutier-Delgosha, O., "Evaluation of ensemble methods for quantifying uncertainties in steady-state CFD applications with small ensemble sizes," Comput. Fluids 203, 104530 (2020).

Zhang, Z., Song, X.-d., Ye, S.-r., Wang, Y.-w., Huang, C.-g., An, Y.-r., and Chen, Y.-s., "Application of deep learning method to Reynolds stress models of channel flow based on reduced-order modeling of DNS data," J. Hydrodyn. 31, 58-65 (2019b).

Zhou, Z., He, G., Wang, S., and Jin, G., "Subgrid-scale model for large-eddy simulation of isotropic turbulent flows using an artificial neural network," Comput. Fluids 195, 104319 (2019).

Zhu, L., Zhang, W., Kou, J., and Liu, Y., "Machine learning methods for turbulence modeling in subsonic flows around airfoils," Phys. Fluids 31, 015105 (2019). 